\title{
Comprehensive Analysis and Expression Profiling of PIN, AUX/LAX, and ABCB Auxin Transporter Gene Families in Solanum tuberosum under Phytohormone Stimuli and Abiotic Stresses
}

\section{Chenghui Yang}

Northwest A\&F University: Northwest Agriculture and Forestry University

\section{Dongdong Wang}

Northwest A\&F University: Northwest Agriculture and Forestry University

Chao Zhang

Northwest A\&F University: Northwest Agriculture and Forestry University

Minghui Ye

Northwest A\&F University: Northwest Agriculture and Forestry University

Nana Kong

Northwest A\&F University: Northwest Agriculture and Forestry University

Haoli Ma

Wuhan University Zhongnan Hospital

Qin Chen ( $\nabla$ chenpeter2289@nwafu.edu.cn)

Northwest A\&F University https://orcid.org/0000-0001-6102-5867

Research article

Keywords: potato, auxin transporter, PIN, AUX/LAX, ABCB, abiotic stress

Posted Date: November 24th, 2020

DOl: https://doi.org/10.21203/rs.3.rs-112829/v1

License: (9) (i) This work is licensed under a Creative Commons Attribution 4.0 International License.

Read Full License 
1 Comprehensive Analysis and Expression Profiling of PIN, AUX/LAX,

4 Chenghui Yang ${ }^{1}$, DongdongWang ${ }^{1}$, Chao Zhang ${ }^{1}$, Minghui Ye ${ }^{1}$, Nana 5 Kong ${ }^{1}$, Haoli $\mathrm{Ma}^{2 *}$, and Qin Chen ${ }^{3 *}$

61 State Key Laboratory of Crop Stress Biology for Arid Areas, College of

7 Agronomy, Northwest A\&F University, Yangling 712100, China

and ABCB Auxin Transporter Gene Families in Solanum tuberosum under Phytohormone Stimuli and Abiotic Stresses

2 Department of Biological Repositories, Zhongnan Hospital of Wuhan University, Wuhan 430071, China

3 College of Food Science and Engineering, Northwest A\&F University, Yangling 712100, China

*Correspondence:mahaoli@nwsuaf.edu.cn(H.M.); chenpeter2289@nwafu. edu.cn (Q.C.)

\section{Abstract}

Background:The phytohormone auxin is known to regulate various aspects of plant growth and development as well as adaptation to environmental stimuli. It is the only plant hormone that exhibits transport polarity that is mediated by three classes of proteins encoded by three families, AUXIN RESISTANT 1/LIKE AUX1 (AUX/LAX) influx carriers, the PIN-FORMED (PIN) efflux carriers, and the ATP-binding cassette B/Multidrug-resistance/P-glycoprotein (ABCB/MDR/PGP) efflux/condition carriers. Extensive studies have been conducted to examine the biological functions of auxin transporter genes using model plants systems and in several plant species. Despite this, information regarding the comprehensive analysis of auxin transporter genes in potato species and information regarding the expression patterns of these genes in response to external stresses remains scarce.

Results: In this study, we conducted a genome-wide annotation of the StLAX, StPIN, and StABCB auxin transporter gene families to examine genomic distributions, gene structures, phylogenic relationships and co-expression analysis. From these analyses, 5 StLAXs, 10 StPINs, and 
$22 \mathrm{StABCBs}$ were identified in the potato genome, and they were mapped to 12 chromosomes. Constructing co-expression networks based on significance changes in gene expression revealed 18 gene modules and potato auxin transporter genes distributed in ten of them correlating to the development of various tissues. Tissue-specific expression analysis of potato auxin transporter genes indicated that genes from the StLAX family were expressed at significantly higher levels compared to those of the other two gene families, suggesting that the StLAXs may be more important for these designated developmental stages in potato. Quantitative real-time PCR (qRT-PCR) analysis indicated responsiveness of StLAXs, StPINs, and StABCBs to auxin and polar auxin transport inhibitors (PATIs), implying their possible roles in mediating intercellular auxin homoeostasis and redistribution. Additionally, the differential expression levels of the StLAX, StPIN, and StABCB genes under abscisic acid (ABA) and abiotic stresses (salt and drought) were indicative of their specific adaptive mechanisms regulating tolerance to various environmental stimuli. Promoter cis-regulatory element analyses were used to explore a large number of auxin-responsive and stress-related cis-elements within the promoters of the StLAX, StPIN, and StABCB genes that could account for their responsiveness to diverse stresses.

Conclusions: In summary, we have provided comprehensive information on StLAX, StPIN, and StABCB auxin transporter gene families in potato. The responsiveness of StLAXs, StPINs, and StABCBs to auxin and PATIs that mediated intercellular auxin homoeostasis and redistribution. Additionally, the differential expression levels of StLAX, StPIN, and StABCB genes in response to ABA and abiotic stresses (salt and drought), suggested that these were specific adaptive mechanisms on tolerance to various environmental stimuli. Promoter cis-regulatory element description analyses suggested that a number of cis-regulatory elements within the promoters of auxin transporter genes in potato targeted by relevant transcription factors to respond to diverse stresses. We are confident that our results provide a foundation for a better understanding of auxin transport in potato, as we have demonstrated the biological 
significance of these family genes in hormone signalling and adaption to environmental stresses.

Keywords: potato, auxin transporter, PIN, AUX/LAX, ABCB, abiotic stress

\section{Background}

Auxin is a plant hormone that possesses multiple functions that regulate the plant growth and development primarily at the cellular level and in response to diverse environmental stimuli as well [1-5]. Auxin is primarily synthesised in leaf primordium, germinating seeds, root tips, and cambiums. The directional polar auxin transport (PAT) system distributes auxin to the targeted tissues to facilitate apical dominance, embryonic development, vascular tissue development, root meristem maintenance, and organ formation and positioning [6]. The cell-cell polar transport of auxin is mediated by proteins localised in the plasma membrane (PM), and these proteins are members of three distinct gene families, the LAX influx carriers [7], the PIN efflux carriers [8, 9], and the $\mathrm{ABCB} / \mathrm{MDR} / \mathrm{PGP}$ efflux/condition transporters [10-12]. Unique polar transport of auxin forms auxin gradients that result in asymmetric distribution of PIN, AUX/LAX, and ABCB proteins across cells and tissues. Regulation of the uneven distribution of auxin within tissues and organs or throughout the entire plant body via auxin transporters provides an important strategy to execute the auxin functions in controlling various plant developmental processes and in response to stressful environments.

AUX/LAX influx carriers encompass four highly conversed family members, and AUX1 and LAX1-3 are characterized as multi membrane-spanning transmembrane proteins that facilitate the entry of auxin into cells [13]. The founder member AUX1 was initially identified from Arabidopsis and primarily functions to promote high-affinity cellular auxin uptake in the root tips [14]. The auxin insensitivel (auxl) mutant exhibits an agravitropic phenotype that is observed in roots after treatment with the auxin influx carrier inhibitor 1-naphthoxyaceticacids (1-NOA), and the gravitropic response in this mutant can be restored by treatment with the membrane-permeable auxin 1-naphthaleneacetic acid 
(NAA) [15, 16]. AtLAX2 participates in the vascular development of cotyledons, and disruption of this gene reinforces cell separation in the quiescent centre (QC) and reduces the expression of the auxin response reporter DR5 $[13,17]$. LAX3 in combination with AUX1, appears to facilitate lateral root development by targeting the auxin-inducible expression of a selection of cell wall remodelling enzymes. The lax 3 mutation in Arabidopsis disrupts lateral root emergence, while the auxllax3 double mutant exhibits a reduced number of emerged lateral roots [18]. The AUX/LAX gene family has also been described in different monocotyledons and dicotyledons in response to hormonal and abiotic stress at the transcriptional level [19-23].

The PIN family is the most extensively studied of the auxin efflux carriers among the auxin transporters in plants, and these carriers play an essential role in PAT [24]. The PIN genes were first cloned in Arabidopsis, where eight members of this gene family were well characterized [25]. Of these genes, several are involved in controlling diverse developmental processes. AtPIN1 is expressed in the vascular bundle of the root, in the inflorescence stem, and the developing organs [26, 27]. Loss-of-function mutations in this gene result in defective floral organs and the formation of naked, pin-shaped inflorescences, the fused leaves, and other shoot abnormalities [9]. Initially, three identified mutant alleles of PIN2 that possessed a strong root agravitropic phenotype were named independently as ethylene-insensitive rootl (eir1), agravitropicl (agr1), and wavy6 (wav6) [28-30]. AtPIN3 is symmetrically located in the root columella cells; however, this protein rapidly re-localises laterally in response to gravity stimulation [31]. Mutations in the Arabidopsis gene PIN3 result in reduced growth and tropic response, and apical hook formation is also altered in pin3 mutants [31]. The functional involvement of AtPIN4 during organogenesis and embryogenesis has been also demonstrated [25, 27]. Disruption of AtPIN4 affects pattern formation in both the embryo and the seedling roots [32]. Therefore, AtPIN4 plays an essential role in auxin maximization and redistribution within the root tip [32]. AtPIN7 is expressed in the basal lineage in the embryo and then later in the root tips, and this protein displays an 
expression pattern that is complementary to that of PIN1 [9]. These loss-of-function phenotypes demonstrate crucial role for these proteins in these developmental processes.

AtPIN5 has been implicated in regulating intracellular auxin homoeostasis and metabolism. pin5 loss- and gain-of-function mutants have been observed to be defective in root and hypocotyl growth [33]. Endoplasmic reticulum (ER)-localization of PIN6 during auxin homoeostasis is required for nectary auxin response, short stamen development, and auxin distribution during root organogenesis in Arabidopsis [34, 35]. Aberrant expression or loss-of-function of PIN6 can interfere with multiple auxin-regulated growth functions, including shoot apical dominance, lateral root primordia development, adventitious root formation, root hair growth, and root waving, indicating that PIN6 acts as a crucial component of auxin homoeostasis [36]. AtPIN8 is preferentially expressed in male gametophytes, with a specific accumulation in pollen from microspore to mature pollen and during pollen germination [37-39]. The effects of loss or gain of function of PIN6, PIN8, and PIN5 are not limited to vein patterning and can extend to the modulation of intracellular auxin response levels [40].

The ATP-binding cassette (ABC) superfamily is a large and diverse group $(\mathrm{A}-\mathrm{H})$ of proteins, and over $100 \mathrm{ABC}$ proteins have been identified to date in plants [41]. In the subfamily B (ABCB) that includes homologs of the mammalian MDRs/PGPs, six members of the ABCB transporter family in Arabidopsis (AtABCB1, AtABCB4, AtABCB14, AtABCB15, AtABCB19, and AtABCB21) have been reported to mediate cellular auxin transport or auxin derivatives. Of these transporters, AtABCB1, AtABCB4, and AtABCB19 are the best-characterized [42, 43]. Since the first plant MDR-like gene (AtABCB1/PGP1/MDR1) was cloned from Arabidopsis, it has been reported that AtPGP1 is localised to the PM and that the corresponding gene is expressed in both the root and shoot apex $[44,45]$. Additionally, the loss of AtMDR 1 results in epinastic cotyledons and reduced apical dominance. Thus, researchers have speculated that AtPGP1 may transport a growth-regulating molecule known as indole-3-acetic acid (IAA) from the shoot apex to influence the 
167 distribution of the hormone auxin during plant development [46]. 168 AtABCB19 has been identified as a stable PM protein that mainly 169 localises to the vascular tissues of the hypocotyl and to the stelae of the 170 root [42]. Defects in PGP19 impair basipetal auxin transport and result in 171 abnormalities in the straight growth of hypocotyl [47]. Moreover, 172 ABCB19 coordinated with PIN1 functions to direct auxin flow from the 173 shoot apex to a maximum in roots [48]. AtABCB4/PGP4 is a 174 root-specific transporter that is predominantly expressed during early root 175 development, and the expression of this transporter has been observed 176 within the root elongation zone and lateral root, and during root hair initiation. atpgp4 mutants exhibit several root phenotypes, such as abnormal lateral root initiation, enhanced root hair elongation, and reduced basipetal auxin transport in roots. These phenotypes suggest the direct involvement of AtPGP4 in auxin homoeostasis within the root [49].

Auxin transporter genes have been widely studied throughout the plant kingdom, including monocotyledons such as Oryza sativa, Sorghum bicolor, and Zea mays, and Arabidopsis thaliana, Populus trichocarpa, Citrullus lanatus, Medicago truncatula and Brassica rapa L.etc belonging to dicotyledons as well [10, 19-23, 50-52]. However, a systematic study of auxin transporter genes in potato is lacking. Given the important roles of auxin transporter proteins during plant growth and development and in response to diverse environmental stimuli, we provide the first comprehensive information detailing the StLAX, StPIN, and StABCB auxin transporter gene families in potato, and we systematically analyse their genomic distributions, gene structures, phylogenic relationships, co-expression analysis and expression profiles. In this study, we emphasise the distinctive spatio-temporal expression patterns of putative StLAX, StPIN, and StABCB genes in response to phytohormone stimuli and abiotic stress. Two PATIs were used to screen for candidate members of auxin transporter gene families responsible for auxin transport. Additionally, cis-regulatory element analysis was incorporated to further examine their expression profiling. Our study aims to provide a foundation for the further exploration of the biological functions of auxin transporter genes. 


\section{Results and discussion}

\section{Genome-wide identification of StLAX, StPIN, and StABCB auxin} transporter genes in potato

From the reference genome retrieved from phytozome 12.1.6 (https://phytozome.jgi.doe.gov/pz/portal.html) for $A$. thaliana, the AtAUX/LAX, AtPIN, and AtABCB protein sequences were used as queries to perform the BLAST searches against the available potato protein sequence data (DM_v3.4_pep_nonredundant) of potato downloaded from PGSC. A total of 5 putative StLAXs and 22 StABCBs were identified from the potato genome, and they were named in accordance with their location order on the chromosomes, with the exception that the nomenclature of 10 StPIN genes was set according to sequence similarity to the $A$. thaliana PIN genes based on previous research [53]. Detailed information regarding $37 \mathrm{~S}$. tuberosum putative auxin transporter-encoding genes, including gene names, locus IDs, open reading frame (ORF) lengths, exon numbers, chromosome locations, deduced polypeptide basic parameters, transmembrane helices, and subcellular localisation predictions, are provided in Table $\mathbf{1 .}$

The deduced StLAX proteins ranged from 468 (StLAX5) to 494 (StLAX1) amino acids in length, and it possessed a molecular weights (MW) ranging from $53.20 \mathrm{kDa}($ StLAX5) to $55.71 \mathrm{kDa}$ (StLAX1) and isoelectric points (pI) between 7.9 (StLAX4) and 8.8 (StLAX5) (Table 1). The prediction for StLAX proteins in regard to their subcellular localisation provides a foundation for further functional research. StLAX1 was predicted to be localised within the cytoplasm, while StLAX2-5 were predicted to be plasma membrane-localized. Furthermore, topology analysis conducted using TMHHM v.2.0 revealed that all of the StLAX proteins possessed 10 transmembrane helices, indicating that their core regions were highly conserved (Table 1, Figure S1 A). Notably, membrane protein StLAX1 was predicted to be cytoplasm-localized, which could be supported by the fact that a nearnest paralogue AtLAX2 was unable to be correctly targeted to the PM when ectopically expressed in epidermal cells [13]. This PM targeting defect of AtLAX2 and StLAX1 
234 might be the requirement of them for additional trafficking factors that 235 performed subfunctionalization in other tissues.

236 The size of the ORF for StPIN proteins varied from 783bp (StPIN8) 237 to $1965 \mathrm{bp}$ (StPIN4). The lengths of the corresponding proteins ranged 238 from 260 to 654 amino acids, and they possessed $28.41 \mathrm{kDa}$ to $71.29 \mathrm{kDa}$ 239 molecular masses and predicted pI values of 6.82 to 9.69 . The number of 240 transmembrane domains for StPIN proteins ranged from 5 to 9. Deletion 241 of a segmental sequence at the C-terminus of the StPIN3 proteins resulted 242 in the depletion of transmembrane helices (Table 1, Figure S1 B). Four 243 StPIN proteins (StPIN2, StPIN5, StPIN6, and StPIN8) were putatively 244 localized within the PM, while StPIN1, StPIN7, and StPIN9 were 245 localized within chloroplasts, and StPIN3 were localized within the 246 cytoplasm. Specifically, StPIN4 was predicted to be localised both in the 247 chloroplasts and the PM, and StPIN10 was predicted to be 248 vacuolar-localized. Various subcellular localisation patterns may be 249 indicative of the specific functions of the PIN gene family.

250 Table 1. Information on StLAX, StPIN, and StABCB genes and properties of the 251 deduced proteins in potato (S. tuberosum). 


\begin{tabular}{|c|c|c|c|c|c|c|c|c|c|}
\hline \multirow[b]{2}{*}{ Gene $^{a}$} & \multirow[b]{2}{*}{ Locus ID ${ }^{\mathbf{a}}$} & \multirow{2}{*}{$\begin{array}{c}\text { ORF } \\
\text { length } \\
(\text { bp) })^{\mathrm{a}}\end{array}$} & \multirow{2}{*}{$\begin{array}{c}\text { No.of } \\
\text { extrons }\end{array}$} & \multirow[b]{2}{*}{ Chromosome Location (bp) ${ }^{\mathrm{a}}$} & \multicolumn{3}{|c|}{ Deducted polypeptid $^{\mathrm{b}}$} & \multirow{2}{*}{$\begin{array}{c}\text { No. of } \\
\text { transmembrane }\end{array}$} & \multirow{2}{*}{$\begin{array}{l}\text { Subcellular } \\
\text { localization }\end{array}$} \\
\hline & & & & & Length (aa) & MI wt (Da) & pI & & \\
\hline StLAX1 & PGSC0003DMT400004027 & 1485 & 8 & ch01:87287332..87290998 (-) & 494 & 55706.95 & 8.5797 & 10 & cyto \\
\hline StLAX2 & PGSC0003DMT400021923 & 1446 & 7 & ch09:210800..214038 (-) & 481 & 54328.39 & 8.1707 & 10 & plas \\
\hline StLAX3 & PGSC0003DMT400059693 & 1467 & 8 & ch10:46747590..46751792 (-) & 488 & 54971.25 & 8.7078 & 10 & plas \\
\hline StLAX4 & PGSC0003DMT400049377 & 1458 & 7 & ch10:50480526..50485783 (-) & 485 & 54606.6 & 7.8706 & 10 & plas \\
\hline StLAX5 & PGSC0003DMT400016760 & 1407 & 8 & ch11:10042232..10046787 (-) & 468 & 53199.12 & 8.7862 & 10 & plas \\
\hline StPIN1 & PGSC0003DMT400014752 & 1845 & 6 & ch03:58350702..58354249(+) & 614 & 67134.08 & 9.3381 & 8 & chlo \\
\hline StPIN2 & PGSC0003DMT400048251 & 1896 & 7 & $\operatorname{ch} 07: 2647114 . .2649884(+)$ & 631 & 68666.48 & 9.4981 & 9 & plas \\
\hline StPIN3 & PGSC0003DMT400015267 & 1395 & 6 & ch04:2170473..2172957 (-) & 464 & 50223.21 & 6.8243 & 5 & cyto \\
\hline StPIN4 & PGSC0003DMT400078330 & 1965 & 6 & $\operatorname{ch} 05: 4250058 . .4253070(-)$ & 654 & 71290.07 & 7.3826 & 9 & chlo/plas \\
\hline StPIN5 & PGSC0003DMT400046253 & 1068 & 5 & $\operatorname{ch} 01: 64013966 . .64017139(-)$ & 355 & 39264.82 & 9.2151 & 9 & plas \\
\hline StPIN6 & PGSC0003DMT400079013 & 1587 & 7 & ch06:41187368..41193747 (-) & 528 & 57652.42 & 8.8456 & 9 & plas \\
\hline StPIN7 & PGSC0003DMT400072459 & 1764 & 6 & ch10:57054506..57057784 (+) & 587 & 63906.07 & 8.8949 & 8 & chlo \\
\hline StPIN8 & PGSC0003DMT400003569 & 783 & 4 & ch02:46450539..46452728 (+) & 260 & 28407.25 & 9.6913 & 5 & plas \\
\hline StPIN9 & PGSC0003DMT400021600 & 1785 & 6 & ch10:59284672..59287550 (-) & 594 & 64304.33 & 9.4377 & 9 & chlo \\
\hline StPIN10 & PGSC0003DMT400027309 & 966 & 3 & ch04:49481160..49482767 (+) & 321 & 35909.45 & 7.2746 & 9 & vacu \\
\hline StABCB1 & PGSC0003DMT400007960 & 3789 & 12 & ch02:30568338..30574681 (+) & 1262 & 136961.15 & 7.3521 & 9 & plas \\
\hline StABCB2 & PGSC0003DMT400003590 & 3750 & 10 & ch02:46284463..46291628(+) & 1249 & 136236.51 & 8.0288 & 9 & plas \\
\hline StABCB3 & PGSC0003DMT400003546 & 3792 & 7 & ch02:46615100..46621182(+) & 1263 & 137488.42 & 8.0619 & 11 & plas \\
\hline StABCB4 & PGSC0003DMT400034908 & 3780 & 12 & ch03:822878..830598 (+) & 1259 & 136332.61 & 8.7596 & 10 & plas \\
\hline StABCB5 & PGSC0003DMT400048379 & 3414 & 8 & ch03:37579500..37585781 (+) & 1137 & 124433.16 & 8.2246 & 9 & plas \\
\hline StABCB6 & PGSC0003DMT400063067 & 1917 & 17 & ch03:55025184..55031533(+) & 638 & 68411.61 & 8.8478 & 6 & plas \\
\hline StABCB7 & PGSC0003DMT400058977 & 4584 & 11 & $\operatorname{ch} 03: 61365905 . .61372730(+)$ & 1527 & 167888.19 & 9.0608 & 12 & plas \\
\hline StABCB8 & PGSC0003DMT400018820 & 3864 & 12 & $\operatorname{ch} 06: 336919 . .342710(+)$ & 1287 & 138575.71 & 7.9275 & 9 & plas \\
\hline StABCB9 & PGSC0003DMT400018812 & 3639 & 10 & $\operatorname{ch} 06: 344414 . .349662(+)$ & 1212 & 130723.67 & 7.799 & 8 & plas \\
\hline StABCB10 & PGSC0003DMT400027962 & 3765 & 7 & ch05:11042954..11047763 (-) & 1254 & 137231.31 & 9.1918 & 11 & plas \\
\hline StABCB11 & PGSC0003DMT400069516 & 3561 & 9 & ch06:53569963..53576584 (+) & 1186 & 130633.57 & 7.4101 & 9 & plas \\
\hline StABCB12 & PGSC0003DMT400013988 & 3681 & 8 & $\operatorname{ch} 07: 11605714 . .11610849(-)$ & 1226 & 134428.48 & 8.6851 & 11 & plas \\
\hline StABCB13 & PGSC0003DMT400049576 & 3780 & 7 & ch07:54289854..54295427 (+) & 1259 & 137937.76 & 9.2144 & 12 & plas \\
\hline StABCB14 & PGSC0003DMT400045176 & 3774 & 12 & ch08:49324870..49334350 (-) & 1257 & 137797.09 & 8.6611 & 10 & plas \\
\hline StABCB15 & PGSC0003DMT400022893 & 1920 & 10 & $\operatorname{ch} 09: 2609568 . .2618204(+)$ & 639 & 69814.41 & 8.7886 & 2 & chlo \\
\hline StABCB16 & PGSC0003DMT400009924 & 4002 & 10 & ch09:5129170..5136543 (+) & 1333 & 145939.27 & 7.8141 & 11 & plas \\
\hline StABCB17 & PGSC0003DMT400019156 & 3864 & 14 & ch11:40225813..40235522(+) & 1287 & 141384.58 & 9.5827 & 11 & plas \\
\hline StABCB18 & PGSC0003DMT400019085 & 3885 & 13 & ch11:40240421..40248719(-) & 1294 & 142465.24 & 8.3389 & 12 & plas \\
\hline StABCB19 & PGSC0003DMT400074962 & 2091 & 17 & ch12:13618482..13637049 (-) & 696 & 78066.69 & 8.8706 & 4 & plas \\
\hline StABCB20 & PGSC0003DMT400030345 & 3651 & 7 & ch12:52311070..52319362(+) & 1216 & 133255.89 & 8.8183 & 8 & plas \\
\hline StABCB21 & PGSC0003DMT400030342 & 3096 & 6 & ch12:52333684..52340071 (+) & 1031 & 112725.71 & 8.785 & 9 & plas \\
\hline StABCB22 & PGSC0003DMT400011930 & 3864 & 12 & ch12:59696990..59702667 (-) & 1287 & 139652.46 & 7.1317 & 11 & plas \\
\hline
\end{tabular}


a, gene information was retrieved from the $S$. tuberosum v4.03 genome annotation (phytozome 12.1.6: http://phytozome.jgi.doe.gov/pz/portal.html)

b, protein profiles were calculated using the Pepstats (https://www.ebi.ac.uk/Tools/seqstats/emboss_pepstats/).

c, transmembrane helices were predicted using the TMHHM Server v2.0 (http://www.cbs.dtu.dk/services/TMHMM/).

d, subcellular localization was predicted by WoLF PSORT (http://www.genscript.com/psort/wolf_psort.html). Plas, plasma membrane; cyto, cytoplasm; chlo, chloroplast; vacu, vacuolar.

The protein properties of the StABCB family vary dramatically. These proteins ranged from 638 to 1527 amino acids in length, possessed $68.41 \mathrm{kDa}$ to $167.89 \mathrm{kDa}$ molecular masses, and exhibited $\mathrm{pI}$ values of 7.35 to 9.58. Additionally, their ORF lengths ranged from 1917bp to $4584 \mathrm{bp}$. For most StABCBs, the conversed domain topology consisted of 2-12 transmembrane helices distributed at the $\mathrm{N}$ - and $\mathrm{C}$-termini. The majority of StABCBs possessed a common domain composition with the exception of three members (StABCB6, StABCB15, and StABCB19) that were missing one or both domains (Table 1, Figure S1 C). With the exception of StABCB15 that was localised within the chloroplast, all the other StABCBs exhibited a possible localisation to the PM. This implied that these $\mathrm{ABCB}$ proteins may act as basal auxin transporters; however, no specialised research has been performed to elaborate on their concrete functions during plant growth and development.

Chromosomal distribution of $S$. tuberosum LAX, PIN, and ABCB auxin transporter gene families

To visualise the organisation of each auxin transporter gene within the S. tuberosum genome, chromosomal mapping of 5 StLAXs, 10 StPINs, and 22 StABCBs was arranged based on the position of these genes on 12 different chromosomes (Table 1, Figure 1). The 37 genes found to be unevenly distributed, where StLAX1-5 were located in an orderly arrangement on chromosomes $01,09,10$, and 11, respectively. Chromosomes 01, 09, and 11 contained only one StLAX gene each, while StLAX3 and StLAX4 presented together on chromosome 10. For StPINs, 8 out of the $12 S$. tuberosum chromosomes contained StPIN proteins, and 
469 no StPIN genes were located on the other four chromosomes

470 (chromosome 08, 09, 11 and 12). Nearly all of chromosomes with the

471 exception of chromosome 01 and 04 contained StABCB genes, and these

472 included four StABCB genes on chromosomes 03 and 12, three StABCBs

473 on chromosomes 02 and 05, and two on chromosomes 07, 09, and 11.

474 Chromosomes 06 and 08 contained only one StABCB gene each.

475 Previous study on potato genome analysis present evidence for at least

476 two whole-genome duplication events [54]. However, analysis of percent

477 ORF nucleotide and amino acid identities of StLAX, StPIN, and StABCB

478 gene families showed no veritable duplicated gene pair shared over $90 \%$

479 identity in both levels (Table S1, S2, and S3). Additionally, some of the

480 StABCB genes were clustered and three tandem duplicated $A B C B$ loci

481 pairs (StABCB8-StABCB9, StABCB17-StABCB18,

482 StABCB20-StABCB21) were present on chromosomes 05, 11, and 12,

483 respectively. In contrast, neither the StLAX loci nor the StPIN loci were

484 derived from tandem duplication. 


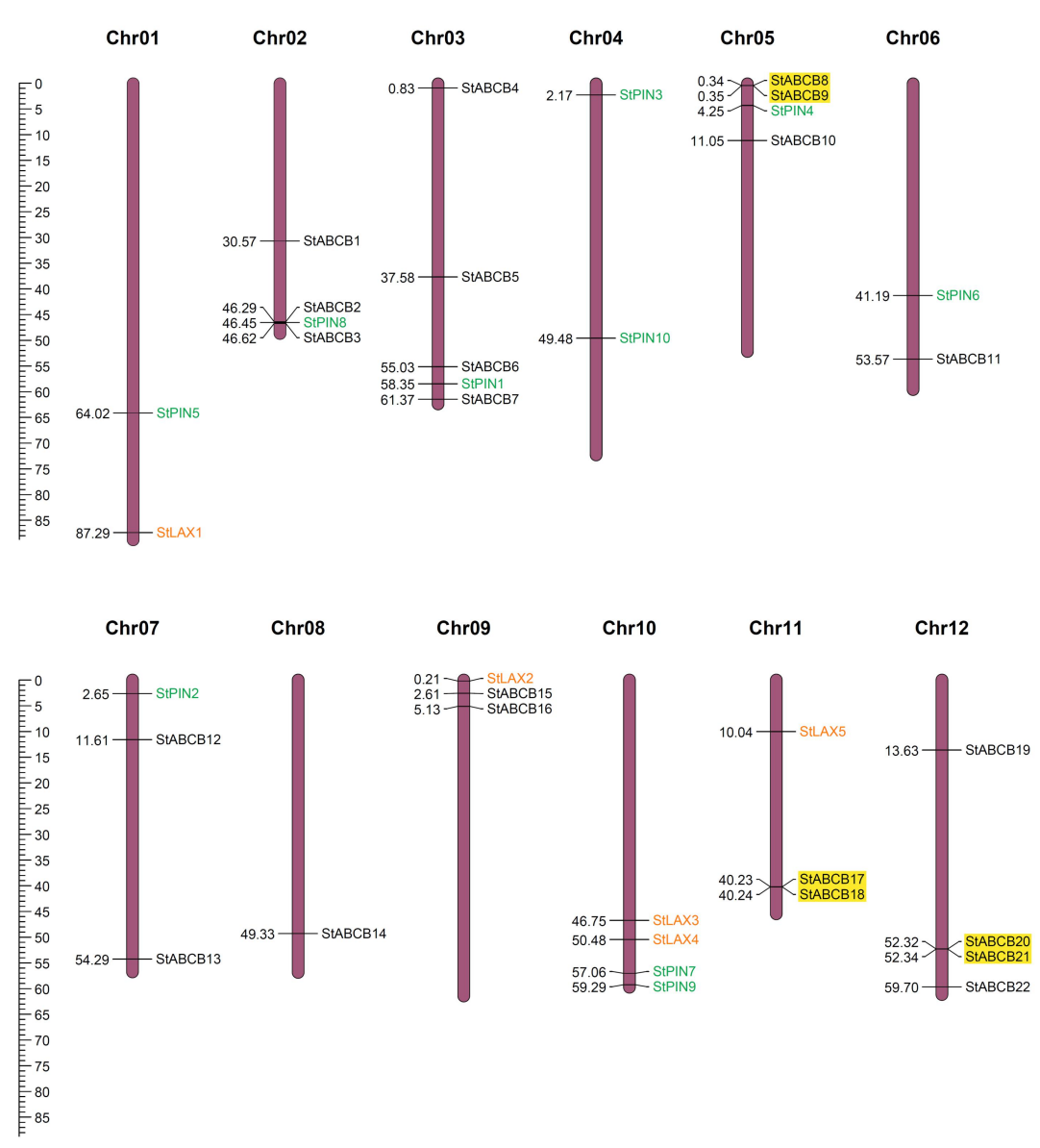

Fig. 1 Chromosomal distribution of StLAX, StPIN, and StABCB family genes. Potato chromosomes were arranged in blocks. Five StLAX genes, 10 StPIN genes and 22 StABCB genes were mapped by locus and the gene clusters were represented by yellow rectangles.

Phylogenetic analysis of LAX, PIN, and ABCB proteins in Arabidopsis, rice, tomato and potato

To date, auxin transporter-encoding gene families in the model plant Arabidopsis have been widely studied for their biological functions in the context of development and responses to the environment [34, 40, 46, 55-57]. And there are increasing evidence of roles auxin transporters play in auxin regulated development in monocotyledon and other dicotyledon species [50, 58]. Therefore, investigation of the phylogenetic relationships of auxin transporter proteins from closely related species including Arabidopsis, rice, tomato and potato will be helpful for understanding the putative biological functions of auxin transporter genes 
in potato. A total of 5 StLAX, 10 StPIN, and 22 StABCB genes, including 4 AtAUX/LAXs, 8 AtPINs, 22 AtABCB proteins (21 transcribed genes and 1 pseudogene) from Arabidopsis, 5 OsLAXs, 12 OsPINs, 22 OsABCBs from rice, and 5 SlLAXs, 10 SlPINs, 29 SlABCBs from tomato were uploaded to construct an unrooted neighbour-joining phylogenetic tree (Figure 2, Table S4). A phylogenetic tree of the LAX proteins revealed two distinct subfamilies, where potato StLAX proteins clustered more closely with the other dicots (tomato and Arabidopsis) compared with those OsLAXs from the monocotyledon species. StLAX2 and StLAX4 proteins were grouped with the closely related SILAX1 and SILAX4 proteins, as they possessed 100\% amino acid identity (Figure 2A). Potato StLAX1 and StLAX3 proteins also shared high homology with Arabidopsis AtLAX2. Moreover, a paralogue gene pair was present between potato StLAX5 and Arabidopsis AtLAX3 proteins.

A previous study performed a detailed analysis of PIN protein structure, and their findings identified two predicted transmembrane domains linked by the central intracellular loop [59]. More precisely, these possessed long loops were defined as canonical PIN proteins, and truly noncanonical PIN proteins possessed shorter loops. These terms gradually replaced the 'long' and 'short' terms that were previously used to describe the structural features of these proteins. The phylogenetic topology structure of the PIN proteins revealed two major clades. The first, which contained the members with the typical canonical structure, was comprised of Arabidopsis AtPIN1 to AtPIN4, and AtPIN7 together with 6 potato PINs (StPIN1-4, StPIN7 and StPIN9), 6 tomato PINs (SIPIN1-4, SIPIN7 and SIPIN9) and four OsPIN1, OsPIN2 and two OsPIN10 proteins. There existed six PIN ortholog gene pairs between potato and tomato, StPIN1-4, StPIN7, StPIN9 and SIPIN1-4, SIPIN7, SIPIN9, respectively. And two paralog gene pairs (StPIN3 with StPIN4 and StPIN7 with StPIN9) existed in the potato PIN gene family. Additionally, four OsPIN1 copies and two OsPIN10 copies showed closer evolutionary relationship, which coincided to the fact that the enlargement of monocot PIN family relied on whole genome duplications and the retention of multiple copies of similar proteins [50] (Figure 2B). 
534 The other clade that possessed the noncanonical AtPIN5 was located on 535 the same branch as StPIN5/SIPIN5, StPIN10/SIPIN10, and OsPIN5, and 536 AtPIN8 was grouped with StPIN8 and SIPIN8 due to high sequence 537 similarity. Additionally, PIN6 is unique among the Arabidopsis PIN 538 proteins due to its dual localisation at the PM and ER, and based on this, 539 it cannot be classified as canonical PIN protein or a noncanonical PIN 540 protein according to the results of the localisation study. In our study, 541 Arabidopsis AtPIN6 and potato StPIN6 proteins constituted a separate clade from the other noncanonical PIN proteins, and this was in accordance with results from a previous publication [35].

A third class of auxin transporters $\mathrm{ABCB}$ was reported to mediate almost all aspects of plant growth and development, and several of these proteins (ABCB1, ABCB4, ABCB14, ABCB15, ABCB19 and $\mathrm{ABCB} 21)$ have been well characterised in regard to their distinct and overlapping functions in A. thaliana [43, 56-57, 60-63]. Phylogenetic analysis of the 95 ABCB proteins from A. thaliana, S. lycopersicum, O. sativa and $S$. tuberosum genomes indicated that the presence of nine clusters, with potato member(s) in the seven groups (Figure 2C). In clade I, AtABCB7 and AtABCB9 were very similar at the sequence level but separate from other ortholog gene pairs (AtABCB3 with AtABCB5, AtABCB11 with AtABCB12 and AtABCB4 with AtABCB21) in Arabidopsis. In particular, there was no $\mathrm{StABCB}$ protein in potato that was clustered with any AtABCBs, while StABCBs exhibited high sequence similarity to SlABCB proteins from tomato. In contrast to clade I, AtABCB2/10, AtABCB13/14, and AtABCB6/20 were deeply branched in clade VI. Meanwhile, StABCB2 and StABCB16 shared significant homology to ABCB19 and ABCB1, respectively, in Arabidopsis, indicating possible roles for StABCB2/16 in auxin transport in plant developmental programs based on close protein sequence similarity to AtABCB19/1. Interestingly, as observed for Arabidopsis ABCBs in clade $\mathrm{V}$, five AtABCB genes (AtABCB15-18 and AtABCB22) subclustered with themselves and a number of the OsABCBs grouped in the same way, suggesting that duplication events occurred within these genes. 

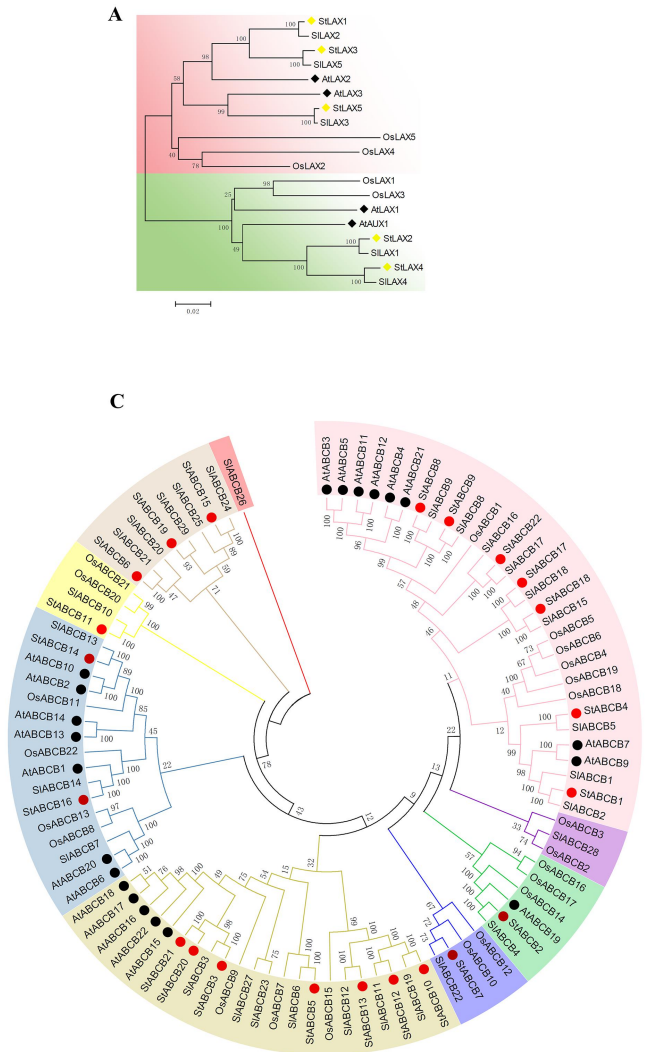

B

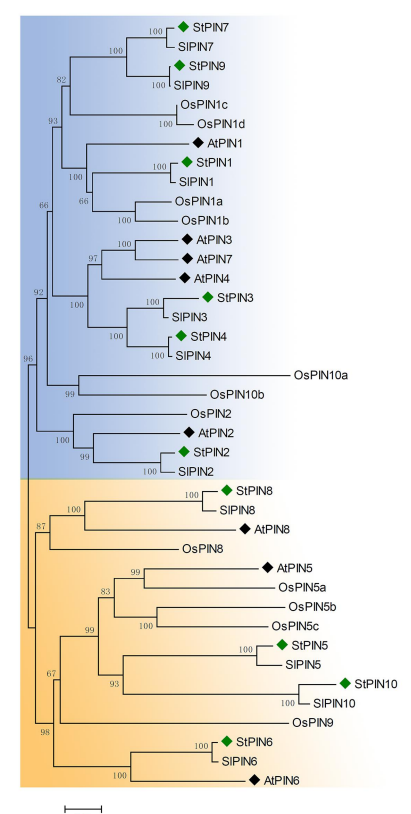

Fig. 2 Phylogenetic tree of LAXs (A), PINs (B), and ABCBs (C) auxin transporter protein families in Arabidopsis, rice, tomato and potato. Bootstrap values were presented for all branches. (A) LAX protein family: inventory of AtLAX families was based on TAIR databases. (B) PIN protein family: sequence data on AtPIN and StPIN families was based on TAIR annotation and Efstathios R's publication[53]. (C) ABCB protein family: inventory of AtABCB families was based on the $\mathrm{ABC}$ superfamily reviewed by Verrier et al[12]. Different colors indicated different subfamilies.

\section{Weighted co-expression network analysis of $\boldsymbol{S}$. tuberosum genes}

A gene expression correlation network was constructed with 25216 differentially expressed genes in various potato tissues by WGCNA method for describing the correlation patterns among genes across microarray samples [64]. A soft thresholding power of 10 with a scale-free model fitting index $\mathrm{R}^{2}>0.688$ was chosen to maximize scale-free topology and maintaining a high mean connectivity (Figure 3A and 3B). Then a dynamic hierarchical tree cut algorithm was constructed to identify stable gene clusters composed of the differentially expressed genes and labelled by unique colors below, including 18 co-expression 
584 modules which were named black (983 genes), blue (2705 genes), 585 darkgreen (4172 genes), darkgrey (448 genes), darkmagenta (253 genes), 586 darkred (2526 genes), darkturquoise (461 genes), green (2354 genes), 587 grey60 (530 genes), magenta (2984 genes), paleturquoise (281 genes), 588 pluml (1016 genes), saddlebrown (289 genes), sienna3 (432 genes), 589 skyblue3 (185 genes), tan (1852 genes), turquoise (3445 genes), violet 590 (265 genes), and 35 probesets were added to the "grey" module for they 591 were not grouped in any of the 18 modules (Figure 3C). Each module expression profile was summarized by a module eigengene (ME), which assembled the most representative gene expression in a module. To understand the consensus modules significance for biological traits, differential expression of the module corresponding eigengenes across the various potato plant tissues was shown as correlation and $p$-values (Figure 3D). Obviously, eigengenes could be characterized by their differential expression profiles in different plant tissues. For example, putative auxin transporter genes in potato investigated in our study were distributed in ten out of 18 consensus modules (Table S5). Furthermore, the construction of the cluster dendrogram among module eigengenes resulted in several meta-modules and the relationship between them were highly preserved (Figure 3E). For example, one meta-module (comprised of the black and darkgrey module eigengenes) represented by StABCB9 and StABCB16 that were differential expressed in leaves. Another meta-module (comprised of the darkgreen and grey 60 module eigengenes) represented by StPIN1/2/3 and StABCB3/22 that tended to be differentially expressed in shoot apex. Moreover, the blue and darkmagenta module eigengenes including StPIN5/6, StABCB1/5/6/8/15 and StLAX4 tended to be co-expressed in stolon. Thus, this analysis revealed that the meta-modules corresponded to a biologically meaningful characterization of modules and genes. 

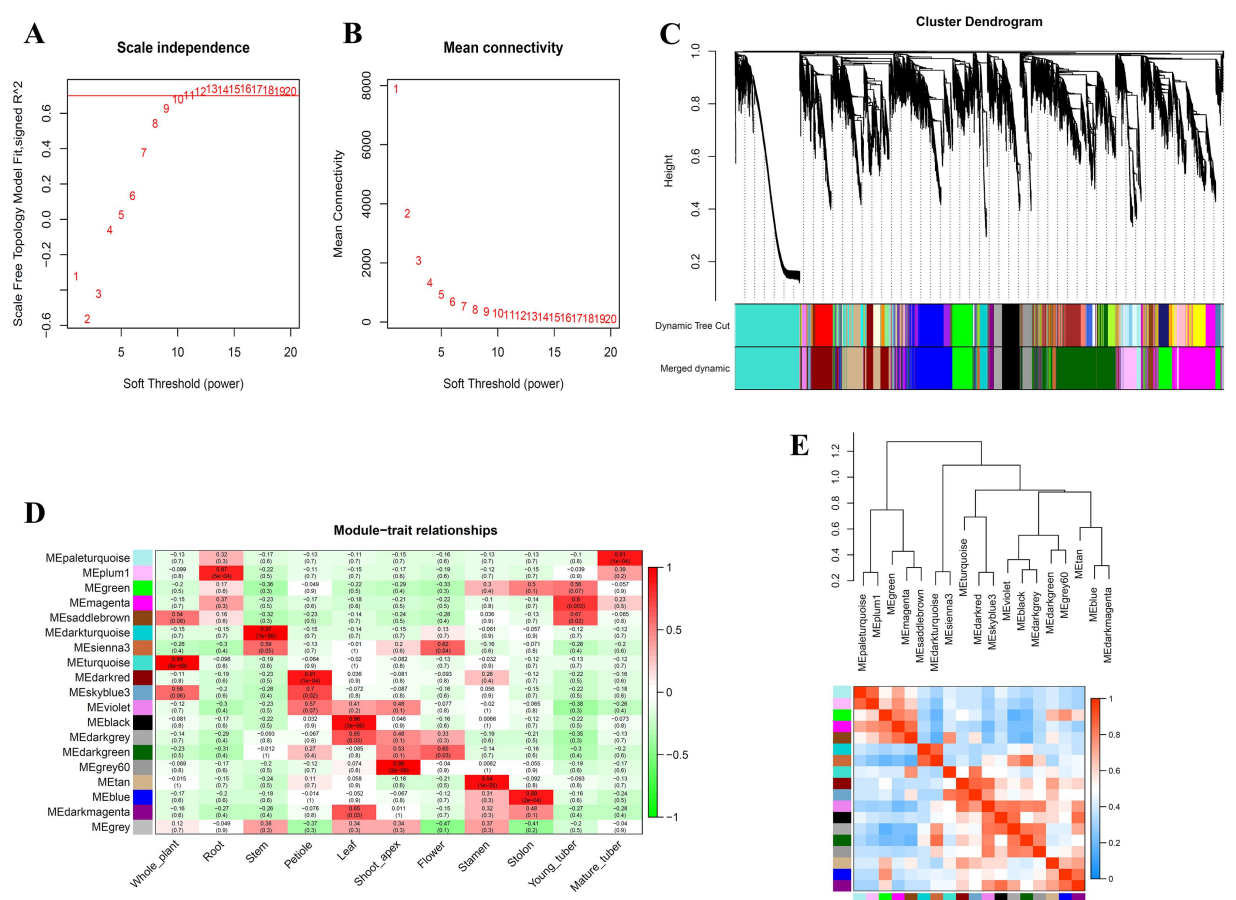

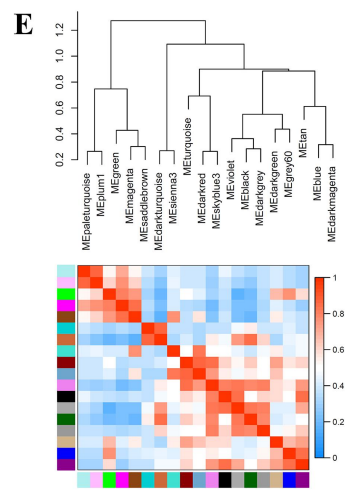

Fig 3. Graphical visualization of the $S$. tuberosum co-expression network. (A) Plot showing the scale free topology $\mathrm{R}^{2}$ value in function of increasing soft thresholding power. (B) Plot showing the relation between mean connectivity and soft threshold. (C) Dendrograms produced by average linkage of hierarchical clustering of $S$. tuberosum genes, which based on a topological overlap matrix (TOM). The modules were assigned colors as indicated in the horizontal bar beneath the dendrogram. (D) Characterizing consensus modules by differential expression of their corresponding eigengenes in the various tissues from potato plant. Red meant over-expression, green meant under-expression; numbers in each cell gave the corresponding t-test $p$-value. Each column corresponded to a tissue and each row corresponded to an eigengene. (E) Clustering dendrograms of consensus module eigengenes for identifying meta-modules (above) and the heatmap for the correlation coefficient between the modules (below). The diagonal plots showed heatmap plots of eigengene adjacencies. Each row and column corresponded to one eigengene (labeled by consensus module color). Within the heatmap, red indicated high adjacency (positive correlation) and green low adjacency (negative correlation) as shown by the color legend.

Gene structure and tissue-specific expression of StLAX, StPIN, and StABCB family genes

To investigate the similarity and diversity in the gene structures of StLAX, StPIN, and StABCB family genes, exon-intron structure analysis 
was performed by comparing the coding sequences with the genomic sequences. The exon number of StLAX genes was either seven or eight, indicating a conversed gene structure (Figure 4, Table 1). From the exon-intron organisation of 10 StPIN genes, the structural patterns of seven canonical type PIN genes (StPIN1-4 and StPIN6-7) were highly conserved with 6-7 exons divided by 5-6 introns, while the number of exons in the other three noncanonical StPIN genes (StPIN5, StPIN8 and StPIN10) varied from 3 to 5 and exhibited a smaller gene size in contrast to these canonical StPINs. In the StABCB gene family, the gene annotation predicted 6 to 17 exons, implying a dramatic variation in the exon-intron structure. This variation in both StPINs and StABCBs was primarily due to divergent intron length, which was one of the predominant factors affecting the gene size.

Analysis of the gene expression profiles in different tissues/organs can be helpful for exploring their possible biological functions. Based on available RNA-seq-generated expression data for potato genotype $\mathrm{RH}$, a heat map was constructed to reveal the expression levels of StLAX, StPIN, and StABCB genes in 8 tissues/organs that included flower, leaf, stem, root, stolon, young tuber, mature tuber, and shoot apex (Figure 4; Table S6). The results revealed that most transcripts from the StLAX, StPIN, and StABCB family genes were detectable in all selected tissues. detection of StABCB10. In the StLAX gene family, StLAX2 was expressed in all tissues at a high level. The transcript levels of StLAX1 were higher in the stolon and exhibited a tissue-specific expression pattern. The remaining StLAX genes were constitutively expressed in all tissues. Additionally, all StLAX genes exhibited the lowest expression in mature tubers. For StPINs, StPIN1, and StPIN4 were ubiquitously expressed, while 5 out of the remaining StPIN genes were preferentially expressed at a specific developmental stage. StPIN2 and StPIN3 were more highly expressed in young tuber than in other organs, and StPIN5 was abundantly expressed in the roots. Meanwhile, two homologous genes, StPIN7 and StPIN9, exhibited relatively high levels in the stolon tissue, indicating that functional redundancy existed among the PIN genes. 
667 Finally, expression analysis of the StABCB gene family revealed that 668 relatively high expression of StABCB2 and StABCB16 was present in all 669 tissues examined, and that StABCB1, StABCB5, and StABCB6 were 670 expressed in a root-preferential manner, implying that these genes may 671 play specific roles in regulating the development of root organs. A 672 number of genes (including StABCB4, StABCB7, StABCB9, StABCB11, 673 StABCB13, StABCB17, StABCB18, and StABCB20-22) exhibited 674 almost no expression or possessed relatively low levels in all the analysed 675 tissues. To summarize these three auxin transporter gene families, genes 676 of the StLAX family were expressed at significantly higher levels than 677 the other two gene families, suggesting that the StLAXs may be more 678 important for these designated developmental stages in potato.

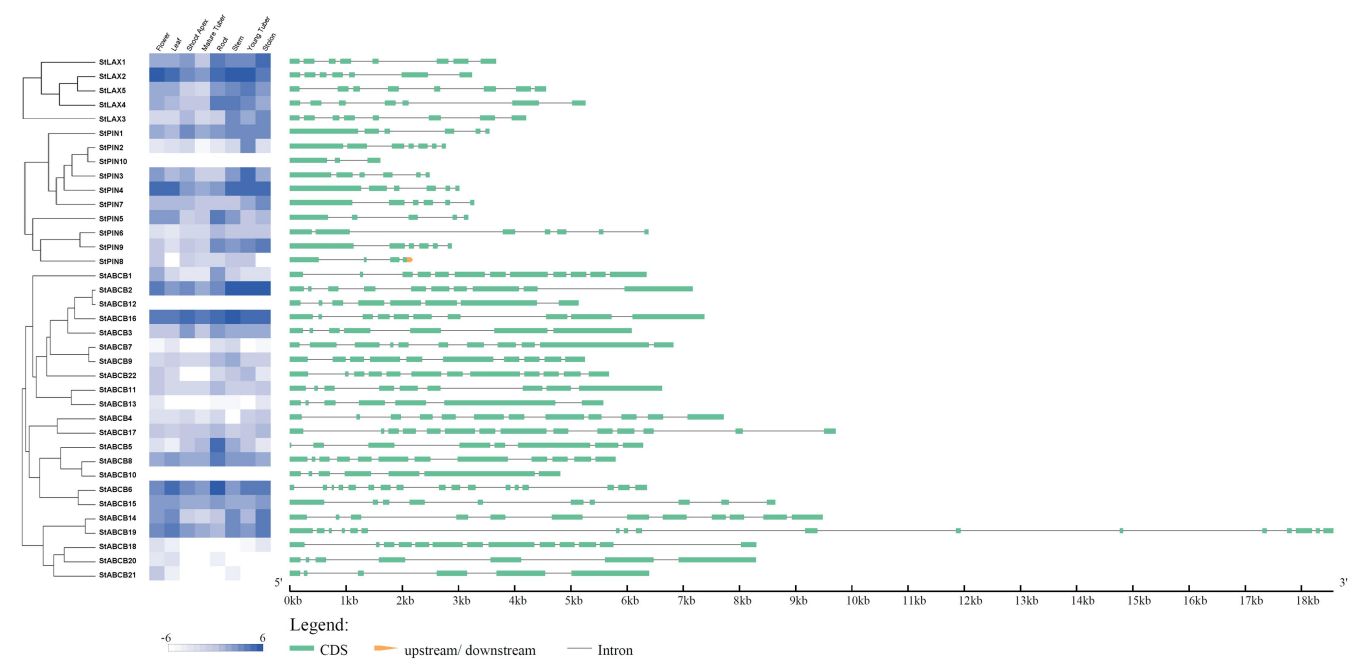

Fig. 4 Tissues-specific expressions and exon-intron structures of StLAX, StPIN, and StABCB genes. The heat map was generated using the Cluster 3.0 software according to the RNA-seq data of RH. The exons were indicated by green boxes and the introns were indicated by gray lines.

\section{Auxin regulation of the StLAX, StPIN, and StABCB genes}

Auxin plays critical roles in regulating plant growth and development via influencing auxin transporters and maintaining intracellular auxin homoeostasis and redistribution [65]. Exogenous IAA application may impact auxin stimulation and transport [32]. To determine how auxin transporters in potato respond to exogenous auxin treatment, we tested the expression levels of members of the StLAX, 
690 StPIN, and StABCB gene families in response to treatment with $10 \mu \mathrm{M}$ 691 IAA for $3 \mathrm{~h}$ using qRT-PCR. The results indicated that most genes were 692 responsive to IAA treatment. In the StLAX family, StLAX1-3 exhibited a 693 lower expression level compared to that of StLAX4, which exhibited a 694 moderate increase. A total of 5 StPIN genes (StPIN1, StPIN2, StPIN5, StPIN7 and StPIN10) were up-regulated, and the remaining StPINs were down-regulated at different levels after IAA treatment. It should be noted that StPIN6 expression in particular strongly decreased. For the StABCB family, the majority of StABCBs exhibited a positive response to auxin, where StABCB1, StABCB3, and StABCB9 sharply upregulated and only 4 genes considerably reduced compared to controls (Figure 5; Table S7). Overall, several StLAX, StPIN, and StABCB genes were up-regulated in response to exogenous IAA, suggesting role for these genes in auxin-related biological progress. There has been little functional characterisation of auxin transporters in potato to date. However, phylogenetic relationships with Arabidopsis could provide clues in regard to the functional identity of auxin transporter genes in potato [21, 51]. In Arabidopsis, the expression of LAX3 was itself auxin-inducible, and LAX3-expressing cells became more efficient sinks for auxin [18]. The paralogue of AtLAX3 in potato, StLAX5, exhibited a downwards trend after auxin treatment and may also be involved in root development. It has been reported that StPIN5 was predominantly expressed in the root [53]. Meanwhile, the functional role of the ortholog gene (AtPIN5) found in A. thaliana in auxin homoeostasis has been confirmed. Therefore, it is possible that StPIN5 functions to maintain auxin homoeostasis in potato based on the close phylogenetic relationship between AtPIN5 and StPIN5, and current evidence suggested that StPIN5 was up-regulated in shoots and down-regulated in roots in response to auxin treatment [66]. In the ABCB family, AtABCB19 was identified as an IAA transporter with strong induction in response to exogenous auxin treatment[11], while the expression of its presumed ortholog in potato, StABCB2, was not upregulated by IAA, suggesting that there exists a variational relationship between these two proteins. This motivated us to explore the role of 
StABCBs in auxin transport. Our findings revealed the existence of a similar feedback mechanism that functioned in regulating the expression of auxin transporter genes in $S$. tuberosum.

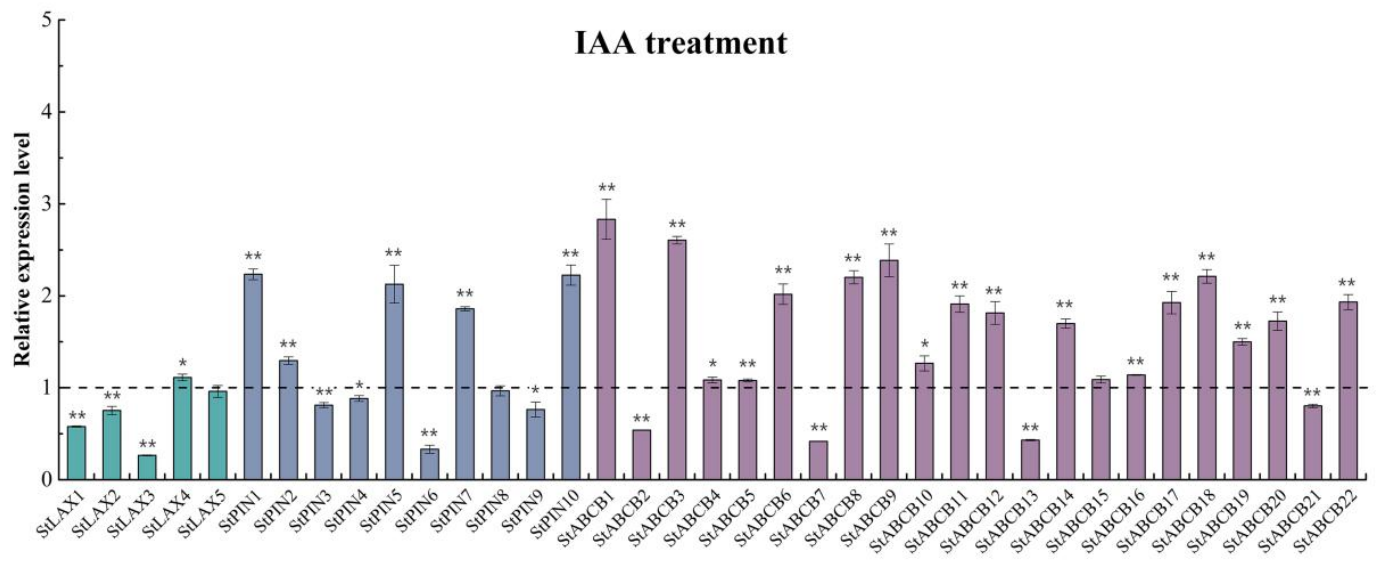

Fig 5. The relative expression values of auxin transporter StLAX, StPIN, and StABCB genes following IAA treatment. Total RNA was extracted from 4-week-old potato plantlets for expression analysis. The histogram represents the relative RNA level of genes after IAA treatment compared with the mock expression level, which was normalized to a value of 1 . The elongation factor 1-a (ef1-a) was employed as the internal standard to normalize the relative mRNA level of individual genes. Error bars represent the standard deviations (SDs) from three biological replicates. (* t-test $P$-value $<0.05$, **t-test $P$-value $<0.01)$.

Expression analysis of StLAX, StPIN, and StABCB genes in response to PATIs treatments

Directional PAT is a form of active intercellular transport mediated by influx and efflux carriers that controls many important plant growth and developmental processes. It is known that PATIs, including auxin influx carrier inhibitor 1-NOA and two auxin efflux carrier inhibitors NPA (1-Naphthylphthalamic acid) and TIBA (2,3,5-triiodobenzoic acid) are major tools that can be used to explore auxin-dependent biological processes. Here, we conducted a PATI assay to investigate the transcriptional fluctuations of StLAX, StPIN, and StABCB under 1-NOA and TIBA treatments to allow us to gain insights into the effects of PATIs on auxin transporters (Figure 6a and 6b; Table S7). Our results revealed that most genes were induced by PATIs. Surprisingly, all StLAX genes were inhibited by 1-NOA due to down-regulation. In contrast, most StPIN and StABCB family genes were insensitive to 1-NOA treatment with the 
750 exception of StABCB1, which was up-regulated dramatically in response 751 to treatment with 1-NOA and of StABCB7, StABCB8, StABCB21 and 752 StABCB22 which exhibited a more moderate increase. For TIBA 753 treatment, most StPIN and StABCB auxin efflux carrier family genes 754 were down-regulated or exhibit slight variations, with the exception of 755 StPIN3 and StPIN9, StABCB8 and StABCB22 that exhibited an 756 increased in response to TIBA. However, the response of StLAX genes to 757 TIBA treatment was irregular, where two of 5 StLAXs were up-regulated 758 and three were down-regulated to varying degrees. Overall, the 759 expression of auxin transporter genes may be blocked by the PATIs. 760 Additionally, we excluded the use of an additionally polar auxin transport 761 inhibitor, NPA, in our present study, as a previous publication had pointed 762 out that NPA was invaluable for demonstrating the involvement of the 763 auxin efflux carrier during PAT-mediated developmental processes [67], 764 and TIBA was sensitive to a greater number of genes than was NPA in 765 regard to auxin response genes in Arabidopsis [68].
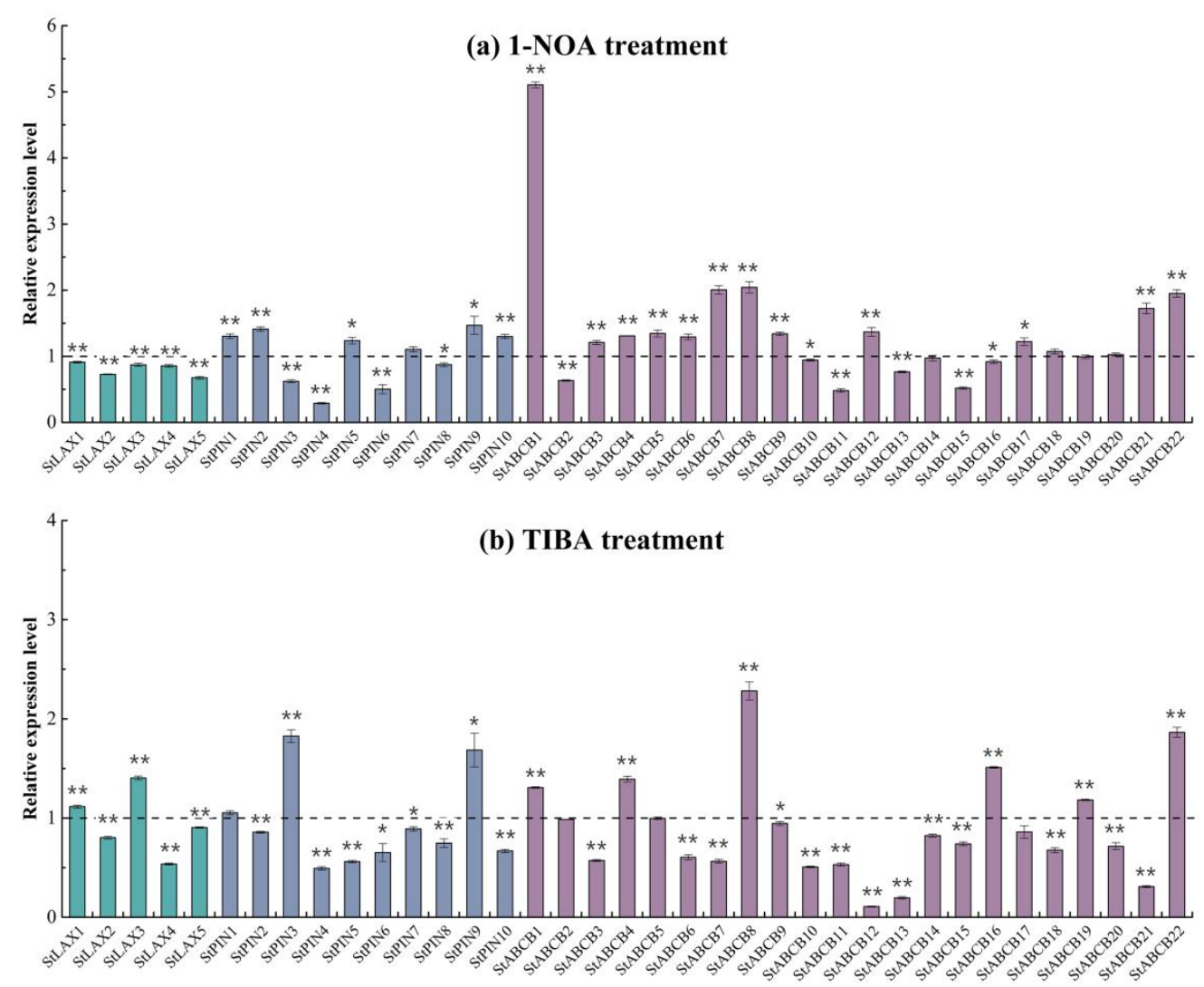
Fig 6. Expression profiles analysis of auxin transporter genes StLAX, StPIN, and StABCB under auxin transport inhibitor treatments. Potato plantlets grown at 4-week-old were treated with $30 \mu \mathrm{M} 1-\mathrm{NOA}$

(a) or $50 \mu \mathrm{M}$ TIBA (b) for $3 \mathrm{~h}$. The relative expression levels were normalized to a value of 1 in the untreated seedlings. Assays were run in triplicates, and bars represent SDs. (* t-test $P$-value $<0.05$, **t-test $P$-value $<0.01)$.

Expression of StLAX, StPIN, and StABCB genes in response to ABA and abiotic stresses

ABA is known as the plant 'stress hormone' and is of predominant significance due to the important role it plays in mediating both biotic and abiotic stress responses in plants [69]. Current evidence has confirmed the specific roles for auxin as a regulator of environmental adaptation in plants [70]. Additionally, the dynamic subcellular trafficking and polarity of PIN proteins are both regulated by a number of environmental responses, thus leading to a complex mechanism that integrates PINs and auxin distribution [8]. To address the possible involvement of $S$. tuberosum auxin transporters in response to abiotic stress at the transcriptional level, qRT-PCR was performed to investigate the expression fluctuations of 37 auxin transporter genes in response to ABA, salinity $(\mathrm{NaCl})$, and drought $(\mathrm{PEG})$ treatment compared to that in untreated plantlets grown in nutrient solution as controls. The data indicated that the majority of genes were responsive to the three stress treatments (Figure 7; Table S8). The majority of StLAX, StPIN, and StABCB genes, with the exception of StPIN2, StPIN5, StPIN8 and StABCB1, StABCB6, StABCB8, StABCB12, StABCB17, and StABCB18, were inhibited by ABA (Figure 7a). In response to salt treatment, 3 of 5 StLAXs (StLAX1, StLAX3, and StLAX5), 4 of 10StPINs (StPIN1, StPIN2, StPIN6, and StPIN9), and 5 of 22 StABCB genes (StABCB4, StABCB8, StABCB9, StABCB16, and StABCB21) were significantly down-regulated compared to levels in the control. In contrast, the rest of the three family genes were all induced by the application of $\mathrm{NaCl}$, and even one or more were strongly up-regulated, including StLAX4, StPIN10, StABCB1, StABCB10, StABCB14, StABCB18, StABCB20, and StABCB22 (Figure 7b). In a similar 
800 manner, half of the $S$. tuberosum auxin transporter genes were repressed 801 by PEG treatment, and most of them were notably down-regulated 802 (Figure 7c). Interestingly, the transcription of several StABCB family 803 genes (StABCB1, StABCB11, StABCB14, StABCB15, StABCB18 and 804 StABCB20) were dramatically up-regulated in response to PEG treatment. 805 Overall, almost all of the $S$. tuberosum auxin transporter genes were 806 transcriptionally mediated by stress hormone ABA, salt, and/or drought 807 treatments, implying that the function of these genes may be associated 808 with abiotic stress responses and adaption. The differential expression 809 profiles of StLAX, StPIN, and StABCB genes indicated that the abiotic 810 stress adaptive mechanism of plants was highly complex. Despite this 811 complexity, a clear relationship between auxin transporter genes and 812 abiotic stresses has been reported in the model plant Arabidopsis. The 813 auxin influx mutant auxl was sensitive to high salt, where stress-induced 814 lateral root elongation was completely blocked [71]. Additionally, salt 815 stress altered the expression and localisation of the PIN2 protein, 816 resulting in reduced gravity response of root growth in Arabidopsis [72]. 817 To date, the effects of hormone and abiotic factors on ABCB gene 818 expression have been reported partially and included the finding that 819 ABA reduced the expression of AtABCB4 and its close homolog 820 AtABCB21 $[60,73]$. The expression of PGP19 was suppressed by the 821 activation of phytochromes and cryptochromes in Arabidopsis [48]. In the 822 present study, we highlighted the responsiveness of StLAX, StPIN, and 823 StABCB auxin transporter genes to hormones and abiotic stresses. Based 824 on this preliminary research, further functional characterisation of each 825 transporter against abiotic stress will be performed using 826 overexpression/knock-out transgenic studies of auxin transporters to help 827 to elucidate the regulatory mechanisms of auxin-abiotic stress signalling. 

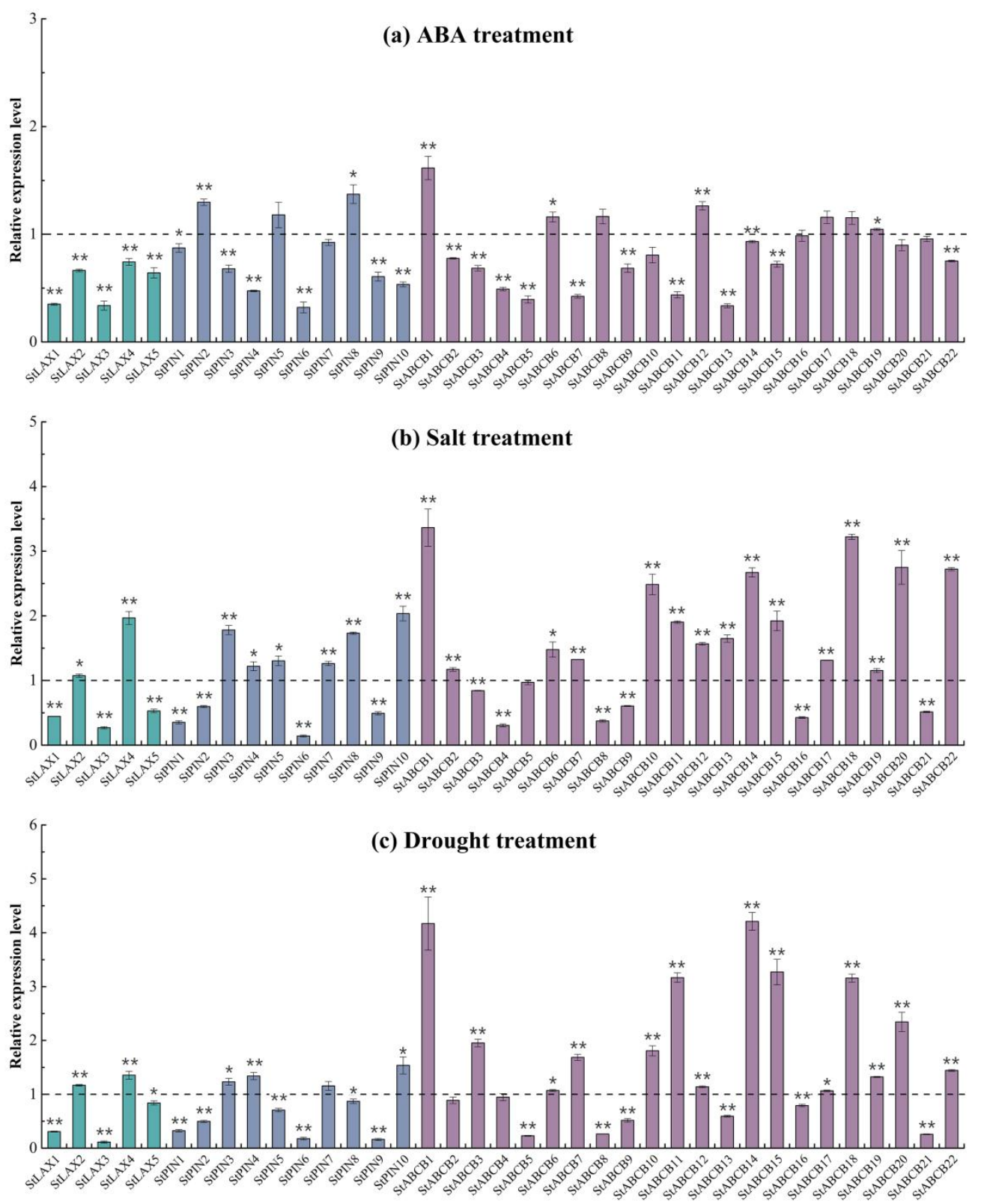

Fig 7. Expression levels of StLAX, StPIN, and StABCB family genes in response to ABA and abiotic stress. Total RNA was extracted from 4-week-old potato plantlets treated with $100 \mu \mathrm{M} \mathrm{ABA}$ (a) for $3 \mathrm{~h}, 200 \mathrm{mM} \mathrm{NaCl}$ (b) or $20 \%$ (W/V) PEG (drought) (c) for 24h. The relative mRNA level of each gene was normalized with respect to the internal reference gene (ef1-a). The data were analyzed by three biological repeats, and SDs were shown with error bars. (* t-test $P$-value $<0.05$, **t-test $P$-value $<0.01)$. gene promoters 
838 decisive role in the transcriptional regulation of their target genes [74]. To 839 identify potential regulatory relationships among auxin transporter genes 840 in potato and cis-regulatory elements located in their promoter regions, 841 we retrieved the putative promoter sequences (2000bp upstream the 842 5'UTR region) of StLAXs, StPINs, and StABCBs from Phytozome $843 \quad 12.1 .6$ for use in scanning of designated cis-regulatory elements. 844 According to the statistical results, a total of 610 auxin-responsive and stress-related cis-regulatory elements were detected in variable numbers (Figure 8; Table S9). Auxin-regulatory cis-elements, including AuxRE (TGTCTC), bZIP response elements (ZREs), Myb response elements (MREs), ABA responsive elements (ABREs), and other abiotic and biotic responsive elements, could account for the response of StLAXs, StPINs, and StABCBs to most external stimuli. Of these, almost all genes in the three families possessed one or more auxin-regulatory cis-elements in their promoter regions, and these elements may be associated with their role in auxin transport. Notably, biotic stress responsive elements (W box) occurred at a high frequency of 2 to 26 sites at each promoter, and this prompted us to speculate that all of $37 \mathrm{~S}$. tuberosum auxin transporter genes may be involved in adaption to biotic stress. Additionally, we found that many auxin transporter genes in potato seemed to have similar cis-elements in their promoter regions while their expression profiles differed from one another. Presumably, there was no correlation between occupancy patterns of cis-elements and gene expression profiles. Even in yeast, Gao et al. confirmed the same conclusion [75]. It could be that the cis-regulatory elements were targeted by several transcription factors such as the ARF binding site (AuxRE:TGTCTC), the WRKYs binding site (W box:TTGAC/TGACT), the bZIPs binding site (ABRE:ACGTG), the bHLHs binding site (MYCR:CACATG), the MYBs binding site (MYBR:CTAACCA), and other homeodomain proteins [76], indicating that a complex regulatory mechanism controls a number of these transcription factors that co-mediate the expression of StLAX, StPIN, and StABCB genes. 


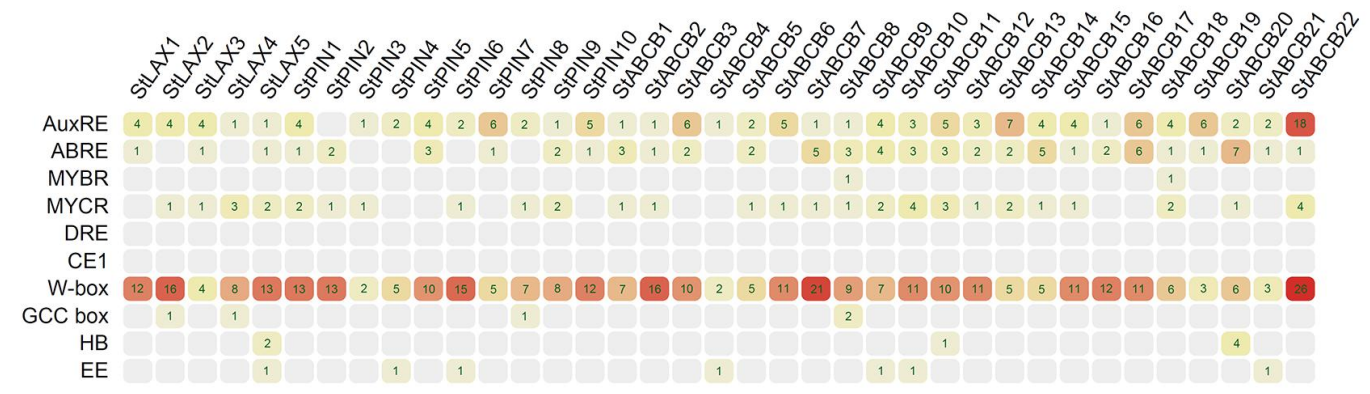

Fig 8. Analysis of auxin-responsive and stress-related cis-regulatory elements in the 2-kb promoter regions of StLAX, StPIN, and StABCB genes.

\section{Conclusions}

In summary, we have provided comprehensive information on StLAX, StPIN, and StABCB auxin transporter gene families in potato, which included basic parameters, chromosomal distribution, phylogeny, co-expression network analysis, gene structure, tissue-specific expression patterns, transcription analysis under exogenous hormone stimuli and abiotic stresses, and cis-regulatory element prediction. The responsiveness of StLAXs, StPINs, and StABCBs to auxin and PATIs that mediated intercellular auxin homoeostasis and redistribution. Additionally, the differential expression levels of StLAX, StPIN, and StABCB genes in response to ABA and abiotic stresses (salt and drought), suggested that these were specific adaptive mechanisms on tolerance to various environmental stimuli. Promoter cis-regulatory element description analyses suggested that a number of cis-regulatory elements within the promoters of auxin transporter genes in potato targeted by relevant transcription factors to respond to diverse stresses. We are confident that our results provide a foundation for a better understanding of auxin transport in potato, as we have demonstrated the biological significance of these family genes in hormone signalling and adaption to environmental stresses.

\section{Methods}

\section{Identification of $\mathrm{AUX} / \mathrm{LAX}$, PIN, and $\mathrm{ABCB}$ auxin transporter}




\section{family genes in potato}

To identify the putative AUX/LAX and ABCB genes in S. tuberosum, the available protein sequence data (DM_v3.4_pep_nonredundant) of potato were downloaded from the Potato Genome Sequencing Consortium (PGSC) [77]. The gene identifier of AtAUX/LAX and AtABCB were obtained from Balzan et al. [50] and sequences of AtAUX/LAX and AtABCB genes retrieved from phytozome 12.1.6 (https://phytozome.jgi.doe.gov/pz/portal.html) were served as queries to perform the Blast searches. Then the Hidden Markov Model (HMM) was used to identify target sequences obtained from the $S$. tuberosum genome. Pfam 01490 (transmembrane amino acid transporter protein) was used for the AUX/LAX family identification and Pfam 00005 (ABC transporter) and Pfam 00664 ( $\mathrm{ABC}$ transporter transmembrane region) were used for the $\mathrm{ABCB}$ family. The remaining sequences were checked for further membrane transport protein domain search using InterProScan Sequence Search (http://www.ebi.ac.uk/Tools/pfa/iprscan/). All identified AUX/LAX and ABCB proteins among $S$. tuberosum genome together with 10 StPIN proteins based on Efstathios R's publication [53] were preserved for downstream analysis. Moreover, their information of molecular weights (MW) and isoelectric points (pI) were calculated by Pepstats (https://www.ebi.ac.uk/Tools/seqstats/emboss_pepstats/). The prediction of the trans-membrane helices for the StLAX, StPIN and StABCB proteins performed by TMHHM v.2.0 (http://www.cbs.dtu.dk/services/TMHMM). And protein subcellular localization was predicted by WoLF PSORT (http://www.genscript.com/psort/wolf_psort.html).

\section{Genome distribution, phylogenetic tree construction, and promoter analysis}

The chromosomal location data of StLAX, StPIN, and StABCB family genes were obtained from phytozome 12.1.6. Distinctive gene names were arranged according to the position from the top to the bottom on chromosomes 1-12. And visualization of chromosome and tandem duplications was employed using the MapChart software. Gene pairs with nucleotide sequence identities over $90 \%$ were considered as duplicated 
928 genes, which were analyzed by DNAMAN software. The alignment 929 contained full-length amino acid sequences of LAX, PIN, and ABCB 930 from Arabidopsis, rice, tomato and potato was generated by ClustalW 931 program with the default parameters and the resulting sequence 932 alignments were then uploaded to construct the the unrooted 933 neighbor-joining tree by the methods of the $p$-distance and complete 934 deletion with a bootstrap of 1,000 replicates using MEGA 6.0 935 (http://www.megasoftware.net/). Additionally, the promoters $(2,000 \mathrm{bp})$ of 936 StLAX, StPIN, and StABCB genes were obtained from Phytozome 12.1.6. And auxin responsive and stress-related cis-regulatory elements analysis were performed using New PLACE (https://www.dna.affrc.go.jp/PLACE/?action=newplace).

\section{Weighted co-expression network construction}

RNA-Seq data from different organs of $S$. tuberosum was chosen to construct a scale-free gene co-expression network. Gene expression levels were normalized to fragments per kilobase per million (FPKM) values. Network analysis was performed using the Weighted Gene Co-expression Network Analysis (WGCNA) R software package following step-by-step network construction and the module detection method. A proper power-law coefficient $\beta$ was selected using the soft-thresholding method, and module stability was tested as the average correlation between the original connectivity and the connectivity from half samples that were randomly sampled 1000 times. The dynamic hierarchical tree cut algorithm was used to identify the co-expression gene modules based on the topological overlap matrix (TOM). Each module was summarized by a module eigengene (ME) through singular value decomposition. Additionally, a permutation test $p$-value was performed to estimate the correlation of differential expression of their corresponding eigengenes in the various tissues from potato plant, and a gene significance (GS) measure could also be defined by minus $\log$ of a $p$-value. Finally, a clustering diagram was plotted with the hclust function in the WGCNA package, and the heatmap for the correlation coefficient between modules was generated using the $\mathrm{R}$ package heatmap.3.R. 


\section{Gene structure and tissue-specific expression profiling analysis}

$$
\text { The Gene Structure Display Server (GSDS) }
$$
(http://gsds.cbi.pku.edu.cn/) was employed to identify exon-intron organizations of StLAX, StPIN, and StABCB family genes by comparing the coding sequences with their corresponding genomic sequences, which were collected from phytozome 12.1.6 (https://phytozome.jgi.doe.gov/pz/portal.html). To characterize the expression patterns of the StLAX, StPIN, and StABCB genes, we used the RNA-Seq data (DM_v4.03) [54] of various tissues of the RH89-039-16 genotype (referred to as RH) including flower, leaves, shoot apex, stolon, young tuber, mature tuber, and root tissue downloaded from PGSC. The expression level was calculated as fragments per kilobase per million (FPKM) values. The raw data of FPKM values were converted as $\log 2$ subsequently and then submitted to HemI [78] for establishing the expression heat maps of hierarchical clustering of the StLAX, StPIN, and StABCB genes.

\section{Plant growth, treatments, and collection of tissues}

The potato cultivar Desiree was used in this study. The plantlets were grown in Murashige and Skoog (MS) medium containing 2\% sucrose and $0.8 \%$ agar and $0.05 \%$ MES (2-Morpholinoethanesulfonic Acid) at $\mathrm{pH}$ 5.8. All plantlets were grown in a plant growth chamber with a $16 \mathrm{~h}$ light $(10000 \mathrm{Lx})$ and $8 \mathrm{~h}$ dark $(0 \mathrm{Lx})$ photoperiod at $22 \pm 1{ }^{\circ} \mathrm{C}$. Then the four-week-old plantlets were transferred into containers of $2 \% \mathrm{MS}$ nutritional liquid medium again and sustained for four weeks under the same growth conditions as before. Potato plantlets with consistent growth vigour were subjected to the phytohormone and abiotic stress treatments. For hormone treatments, four-week-old plantlets were soaked in $2 \% \mathrm{MS}$ nutritional liquid medium with $10 \mathrm{uM}$ IAA, $50 \mathrm{uM}$ TIBA, $30 \mathrm{uM}$ 1-NOA, and $100 \mathrm{uM} \mathrm{ABA}$ then incubated for 3 hours. For stress experiments, the roots of potato plantlets were immersed in nutritional liquid medium containing $200 \mathrm{mM} \mathrm{NaCl}$ or $20 \%$ (W/W) Polyethylene glycol (PEG6000) for 24 hours. Untreated plantlets were used as controls. The whole treated and control potato plantlets were collected for RNA extraction. For each treatment condition, three biological replicates were established to reduce 
996 the error rate and a collection of samples from four potato plantlets were 997 used as one biological replicate.

\section{RNA isolation and qRT-PCR analysis}

Total RNA from whole in vitro-grown plantlets was extracted using the high purity total RNA rapid extraction kit (TIANGEN, Beijing, China) based on manufacturer's instructions. Gel electrophoresis was used to assess RNA quality and quantity. First-strand cDNA was synthesized from 2 ug of total RNA using the Fast Super RT Kit cDNA with gDNase (TIANGEN, Beijing, China). The primers sequences of individual gene families for qRT-PCR analysis were designed with Primer Primer 5 software and confirmed their specificity of unique and appropriate cDNA segments by uploading to the BLAST program (Table S10). qRT-PCR was performed on the Q7 Real Time PCR System using 2xRealStar Green Fast Mixture (GenStar, Beijing, China) with elongation factor 1- $\alpha$ (efl- a ) as the internal reference gene for normalization of gene expression [79]. The reaction was carried out in a total volume of $10 \mu 1$ containing $0.4 \mu \mathrm{L}$ cDNA as template, $5 \mu \mathrm{L}$ RealStar Green Fast Mixture (2x), $0.4 \mu \mathrm{L}$ of each forward and reverse primer $(10 \mu \mathrm{M})$, and RNase-free water up to $10 \mu \mathrm{L}$. In qRT-PCR experiments the following thermal cycling conditions were applied, initial activation $95^{\circ} \mathrm{C}$ for $2 \mathrm{~min}$, then 40 cycles of $95^{\circ} \mathrm{C}$ for $15 \mathrm{~s}, 55^{\circ} \mathrm{C}$ for $15 \mathrm{~s}$ and $72^{\circ} \mathrm{C}$ for $19 \mathrm{~s}$. The melting curve generated from $65^{\circ} \mathrm{C}$ to $95^{\circ} \mathrm{C}$ with increments of $0.5^{\circ} \mathrm{C}$ every $5 \mathrm{~s}$ was performed to check specific amplification. The relative RNA levels of each gene were calculated from cycle threshold $\left(\mathrm{C}_{\mathrm{T}}\right)$ values according to the $2^{-\Delta \Delta C T}$ method [80]. The data were analysed using SPSS software (SPSS version 19.0, SPSS, Chicago, IL, USA), using descriptive statistical tests; one-way analysis of variance was used to evaluate the differences between treatments and control. Statistical significance was established at 0.05 and 0.01 , respectively.

\section{Additional files}

Additional file 1: Fig S1. Transmembrane topology prediction for the StLAX (A), StPIN (B), and StABCB (C) proteins. The trans-membrane were using

the

TMHHM v.2.0 
(http://www.cbs.dtu.dk/services/TMHMM) and the predicted

1030 trans-membrane helices was shown as red peaks.

1031 Additional file 2: Table S1. The primer sequences used in qPCR 1032 analyses.

1033 Additional file 3: Table S2. Percent ORF nucleotide (bottom-left) and 1034 amino acid (up-right, bold) identities of StLAXs.

1035 Additional file 4: Table S3. Percent ORF nucleotide (bottom-left) and 1036 amino acid (up-right, bold) identities of StPINs.

1037 Additional file 5: Table S4. Percent ORF nucleotide (bottom-left) and 1038 amino acid (up-right, bold) identities of StABCBs.

1039 Additional file 6: Table S5. Protein sequences used in the phylogenetic relationship analysis.

Additional file 7: Table S6. Gene modules and significance $p$-value of auxin transporters in potato.

Additional file 8: Table S7. FPKM values of auxin transporter genes in various tissues.

Additional file 9: Table S8. Raw data of auxin transporter genes under IAA and PATIs treatments.

Additional file 10: Table S9. Raw data of auxin transporter genes under ABA and abiotic stresses.

Additional file 11:Table S10. Motif sites of cis-elements within the promoters of auxin transporter genes.

\section{Authors' contributions}

Q.C., H.M. and C.Y. conceived and designed the research plans; D.W., M. Y. and N.K. participated in most of the experiments and data collection; C.Z., M.Y. and N.K. provided technical assistance to C.Y.; C.Y. wrote the manuscript with contributions from all the authors; H.M. and C.Y. revised the manuscript. All authors read, reviewed and approved the final manuscript.

\section{Author details}

1059 State Key Laboratory of Crop Stress Biology for Arid Areas, College of 1060 Agronomy, Northwest A\&F University, Yangling 712100, China 
1061 Department of Biological Repositories, Zhongnan Hospital of Wuhan

1062 University, Wuhan 430071, China

1063 College of Food Science and Engineering, Northwest A\&F University,

1064 Yangling 712100, China

1065 Competing interests

1066 The authors declare no competing interests.

1067 Availability of data and materials

1068 All data generated or analyzed during this study are included in this 1069 published article.

1070 Consent for publication

$1071 \quad$ Not applicable.

1072 Ethics approval and consent to participate

$1073 \quad$ Not applicable.

1074 Funding

1075 This work was mainly funded by the National Key Research and 1076 Development Program of China (2018YFD0200805), the Key 1077 Technology Development Program of Science and Technology 1078 Department of Shaanxi province (2017ZDXM-NY-004) and partially 1079 supported by State Key Laboratory of Crop Stress Biology in Arid Areas, 1080 China.

1081

1082

1083

1084

1085

1086

1087

1088

1089

Conflict of Interest

None of the authors have any actual or potential conflicts of interest.

\section{Acknowledgments}

The authors would like to acknowledge Gang Li, Department of Biological Repositories, Zhongnan Hospital of Wuhan University for providing technical assistance to us.

\section{Endnotes}

[1] https://phytozome.jgi.doe.gov/pz/portal.html

[2] https://www.ebi.ac.uk/Tools/seqstats/emboss_pepstats/ 
1090

1091

1092

1093

1094

[3] http://www.cbs.dtu.dk/services/TMHMM/

[4] http://www.genscript.com/psort/wolf_psort.html

[5] http://www.megasoftware.net/

[6] https://www.dna.affrc.go.jp/PLACE/?action=newplace

[7] http://gsds.cbi.pku.edu.cn/

\section{References}

1. Dubrovsky, J. G., Sauer, M., Napsucialy-Mendivil, S., Ivanchenko, M. G., Friml, J., Shishkova, S., et al. Auxin acts as a local morphogenetic trigger to specify lateral root founder cells. Proc. Natl. Acad. Sci. U. S. A. 2008;105(25):8790-4.

2. Gallavotti, A. The role of auxin in shaping shoot architecture. J. Exp. Bot. 2013;64(9):2593-608.

3. Novak, S. D., Luna, L. J., and Gamage, R. N. Role of auxin in orchid development. Plant Signal. Behav. 2014;9(10), e972277.

4. Rahman, A. Auxin: a regulator of cold stress response. Physiol. Plant. 2013;147(1):28-35.

5. Ghanashyam, C., and Jain, M. Role of auxin-responsive genes in biotic stress responses. Plant Signal. Behav. 2009;4(9):846-8.

6. Ljung, K., Hull, A. K., Kowalczyk, M., Marchant, A., Celenza, J., Cohen, J. D., et al. Biosynthesis, conjugation, catabolism and homeostasis of indole-3-acetic acid in Arabidopsis thaliana. Plant Mol.Biol. 2002;50(2):309-32.

7. Ranjan, S., and Benjamin, P. AUX/LAX family of auxin influx carriers-an overview. Front. Plant Sci. 2012;3:225.

8. Adamowski, M., and Friml, J. PIN-dependent auxin transport: action, regulation, and evolution. Plant Cell 2015;27(1):20-32.

9. Krecek, P., Skupa, P., Libus, J., Naramoto, S., Tejos, R., Friml, J., et al. The PIN-FORMED (PIN) protein family of auxin transporters. Genome Biol. 2009;10:249.

10. Yang, H., and Murphy, A. S. Functional expression and characterization of Arabidopsis ABCB, AUX1 and PIN auxin 
transporters in Schizosaccharomyces pombe. Plant J. 2010;59(1):179-91.

11. Geisler, M., and Murphy, A. S. The ABC of auxin transport: the role of p-glycoproteins in plant development. FEBS Lett. 2006;580(4):1094-102.

12. Verrier, P. J., Bird, D., Burla, B., Dassa, E., Forestier, C., Geisler, M., et al. Plant ABC proteins-a unified nomenclature and updated inventory. Trends Plant Sci. 2008;13(4):151-9.

13. Péret, B., Swarup, K., Ferguson, A., Seth, M., Yang, Y., Dhondt, S., et al. AUX/LAX genes encode a family of auxin influx transporters that perform distinct functions during Arabidopsis development. Plant Cell 2012;24(7):2874-85.

14. Yang, Y., Hammes, U. Z., Taylor, C. G., Schachtman, D. P., and Nielsen, E. High-affinity auxin transport by the AUX1 influx carrier protein. Curr. Biol. 2006;16(11):1123-7.

15. Swarup, R. Localization of the auxin permease AUX1 suggests two functionally distinct hormone transport pathways operate in the Arabidopsis root apex. Genes Dev. 2001;15(20):2648-53.

16. Swarup, R. Structure-function analysis of the presumptive Arabidopsis auxin permease AUX1. Plant Cell 2004;16(11):3069-83.

17. Zhang, W., Swarup, R., Bennett, M., Schaller, G. E., and Kieberet, J. J. Cytokinin induces cell division in the quiescent center of the Arabidopsis root apical meristem. Curr. Biol. 2013;23(20):1979-89.

18. Swarup, K., Benková, E., Swarup, R., Casimiro, I., Péret, B., Yang, Y., et al. The auxin influx carrier LAX3 promotes lateral root emergence. Nat. Cell Biol. 2008;10(8):946-54.

19. Yue, R., Tie, S., Sun, T., Zhang, L., Yang, Y., Qi, J., et al. Genome-wide identification and expression profiling analysis of ZmPIN, ZmPILS, ZmLAX and ZmABCB auxin transporter gene families in maize (Zea mays L.) under various abiotic stresses. PLoS One 2015;10(3), e0118751.

20. Shen, C., Bai, Y., Wang, S., Zhang, S., Wu, Y., Chen, M., et al. Expression profile of PIN, AUX/LAX and PGP auxin transporter gene families in Sorghum bicolor under phytohormone and abiotic 
stress. FEBS J. 2010;277(14):2954-69.

21. Carraro, N., Tisdaleorr, T. E., Clouse, R. M., Anne, S. K., and Rachel, S. Diversification and expression of the PIN, AUX/LAX, and ABCB families of putative auxin transporters in Populus. Front. Plant Sci. 2012;3:17.

22. Yu, C., Dong, W., Zhan, Y., Huang, Z. A., Li, Z., Kim, I. S., et al. Genome-wide identification and expression analysis of ClLAX, ClPIN and ClABCB genes families in Citrullus lanatus under various abiotic stresses and grafting. BMC Genet. 2017;18:33.

23. Gao, L. W., Lyu, S. W., Tang, J., Zhou, D. Y., Bonnema, G., Xiao, D., et al. Genome-wide analysis of auxin transport genes identifies the hormone responsive patterns associated with leafy head formation in Chinese cabbage. Sci Rep 2017;7:42229.

24. Sawchuk, M. G., and Scarpella, E. Control of vein patterning by intracellular auxin transport. Plant Signal. Behav. 2013;8(11), e27205.

25. Paponov, I. A., Teale, W. D., Trebar, M., Blilou, I., and Palme, K. The PIN auxin efflux facilitators: evolutionary and functional perspectives. Trends Plant Sci. 2005;10(4):170-7.

26. GäLweiler, L., Guan, C., Müller, A., Wisman, E., Mendgen, K., Yephremov, A., et al. Regulation of polar auxin transport by AtPIN1 in Arabidopsis vascular tissue. Science 1998;282(5397):2226-30.

27. Benková, E., Michniewicz, M., Sauer, M., Teichmann, T., Seifertová, D., Jürgens, G., et al. Local, efflux-dependent auxin gradients as a common module for plant organ formation. Cell 2003;115(5):591-602.

28. Müller, A., Guan, C., Gälweiler, L., Tänzler, P., Huijser, P., Marchant, A., et al. AtPIN2 defines a locus of Arabidopsis for root gravitropism control. EMBO J. 1998;17(23):6903-11.

29. Luschnig, C., Gaxiola, R. A., Grisafi, P., and Fink, G. R. EIR1, a root-specific protein involved in auxin transport, is required for gravitropism in Arabidopsis thaliana. Genes Dev. 1998;12(14):2175-87.

30. Chen, R., Hilson, P., Sedbrook, J., Rosen, E., Caspar, T., and Masson, 
P. H. The Arabidopsis thaliana AGRAVITROPIC1 gene encodes a component of the polar-auxin-transport efflux carrier. Proc. Natl. Acad. Sci. U. S. A. 1998;95(25):15112-7.

31. Friml, J., Wiśniewska, J., Benková, E., Mendgen, K., and Palme, K. Lateral relocation of auxin efflux regulator PIN3 mediates tropism in Arabidopsis. Nature 2002;415(6873):806-9.

32. Friml, J., Benková, E., Blilou, I., Wisniewska, J., and Palme, K. AtPIN4 mediates sink-driven auxin gradients and root patterning in Arabidopsis. Cell 2002;108(5):661-73.

33. Friml, J., Vieten, A., Sauer, M., Weijers, D., Schwarz, H., Hamannet, T., et al. Efflux-dependent auxin gradients establish the apical-basal axis of Arabidopsis. Nature 2003;426(6963):147-53.

34. Mravec, J., Skůpa, P., Bailly, A., Hoyerová, K., Krecek, P., Bielach, A., et al. Subcellular homeostasis of phytohormone auxin is mediated by the ER-localized PIN5 transporter. Nature 2009;459(7250):1136-40.

35. Simon, S., Skůpa, P., Viaene, T., Zwiewka, M., Tejos, R., Klíma, P., et al. PIN6 auxin transporter at endoplasmic reticulum and plasma membrane mediates auxin homeostasis and organogenesis in Arabidopsis. New Phytol. 2016;211(1):65-74.

36. Bender, R. L., Fekete, M. L., Klinkenberg, P. M., Hampton, M., Bauer, B., Malecha, M., et al. PIN6 is required for nectary auxin response and short stamen development. Plant J. 2013;74(6):893-904.

37. Nisar, N., Cuttriss, A. J., Pogson, B. J., and Cazzonelli, C. I. The promoter of the Arabidopsis PIN6 auxin transporter enabled strong expression in the vasculature of roots, leaves, floral stems and reproductive organs. Plant Signal. Behav. 2014;9(1), e27898.

38. Honys, D., and Twell, D. Transcriptome analysis of haploid male gametophyte development in Arabidopsis. Genome Biol. 2004;5(11), R85.

39. Pina, C., Pinto, F., Feijó, J. A., and Becker, J. D. Gene family analysis of the Arabidopsis pollen transcriptome reveals biological implications for cell growth, division control, and gene expression regulation. Plant Physiol. 2005;138(2):744-56.

40. Ding, Z., Wang, B., Moreno, I., Dupláková, N., Simon, S., Carraro, 
N., et al. ER-localized auxin transporter PIN8 regulates auxin homeostasis and male gametophyte development in Arabidopsis. Nat. Commun. 2012;3:941.

41. Sawchuk, M. G., Alexander, E., and Enrico, S. Patterning of leaf vein networks by convergent auxin transport pathways. PLoS Genet. 2013;9(2), e1003294.

42. Theodoulou, F. L. Plant ABC transporters. Biochimica et Biophysica Acta 2000;1465(1):79-103.

43. Cho, M., and Cho, H. T. The function of $\mathrm{ABCB}$ transporters in auxin transport. Plant Signal. Behav. 2013;8(2), e22990.

44. Titapiwatanakun, B., and Murphy, A. S. Post-transcriptional regulation of auxin transport proteins: cellular trafficking, protein phosphorylation, protein maturation, ubiquitination, and membrane composition. J. Exp. Bot. 2009;60(4):1093-107.

45. Dudler, R., and Hertig, C. Structure of an mdr-like gene from Arabidopsis thaliana. Evolutionary implications. J. Biol. Chem. 1992;267(9):5882-8.

46. Sidler, M., Hassa, P., Hasan, S., Ringli, C., and Dudleret R. Involvement of an $\mathrm{ABC}$ transporter in a developmental pathway regulating hypocotyl cell elongation in the light. Plant Cell 1998;10(10):1623-36.

47. Noh, B., Murphy, A. S., and Spalding, E. P. Multidrug resistance-like genes of Arabidopsis required for auxin transport and auxin-mediated development. Plant Cell 2001;13(11):2441-54.

48. Nagashima, A., Suzuki, G., Uehara, Y., Saji, K., and Sakai, T. Phytochromes and cryptochromes regulate the differential growth of Arabidopsis hypocotyls in both a PGP19-dependent and a PGP19-independent manner. Plant J. 2008;53(3):516-29.

49. Santelia, D., Vincenzetti, V., Azzarello, E., Bovet, L., Fukao, Y., Düchtig, P., et al. MDR-like ABC transporter AtPGP4 is involved in auxin-mediated lateral root and root hair development. FEBS Lett. 2005;579(24):5399-406.

50. Balzan, S., Johal, G. S., and Carraro, N. The role of auxin transporters in monocots development. Front. Plant Sci. 2014;5:393. 
51. Shen, C., Yue, R., Bai, Y., Feng, R., Sun, T., Wang, X., et al. Identification and analysis of Medicago truncatula auxin transporter gene families uncover their roles in responses to Sinorhizobium meliloti infection. Plant Cell Physiol. 2015;56(10):1930-43.

52. Chai, C., and Subudhi, P. Comprehensive analysis and expression profiling of the OsLAX and OsABCB auxin transporter gene families in rice (Oryza sativa) under phytohormone stimuli and abiotic stresses. Front. Plant Sci. 2016;7:593.

53. Roumeliotis, E., Kloosterman, B., Oortwijn, M., Visser, R. G. F., and Bachem, C. W. B. The PIN family of proteins in potato and their putative role in tuberization. Front. Plant Sci. 2013;4:524.

54. Xu, X., Pan, S., Cheng, S., Zhang, B., Mu, D., Ni, P., et al. The Potato Genome Sequencing Consortium. Genome sequence and analysis of the tuber crop potato. Nature 2011;475(7355):189-95.

55. Vandenbussche, F., Petrasek, J., Zadnikova, P., Hoyerová, K., Pesek, B., Raz, V., et al. The auxin influx carriers AUX1 and LAX3 are involved in auxin-ethylene interactions during apical hook development in Arabidopsis thaliana seedlings. Development 2010;137(4):597-606.

56. Kube, M., Yang, H., Richter, G. L., Cheng, Y., Młodzińska, E., Wang, $\mathrm{X}$, et al. The Arabidopsis concentration-dependent influx/efflux transporter ABCB4 regulates cellular auxin levels in the root epidermis. Plant J. 2012;69(4):640-54.

57. Lewis, D. R., Wu, G., Ljung, K., and Spalding, E. P. Auxin transport into cotyledons and cotyledon growth depend similarly on the ABCB19 multidrug resistance-like transporter. Plant J. 2009;60(1):91-101.

58. Hoyerova, K., Perry, L., Hand, P., Lanková, M., Kocábek, T., May, S., et al. Functional characterization of PaLAX1, a putative auxin permease, in heterologous plant systems. Plant Physiol. 2008;146(3):1128-41.

59. Tom, B., Brockington, S. F., Carl, R., Graham, S. W., Dennis, S., Toni, K., et al. Paralogous radiations of PIN proteins with multiple origins of noncanonical PIN structure. Mol. Biol. Evol. 2014;31(8):2042-60. 
60. Kamimoto, Y., Terasaka, K., Hamamoto, M., Takanashi, K., Fukuda, S., Shitan, N., et al. Arabidopsis ABCB21 is a facultative auxin importer/exporter regulated by cytoplasmic auxin concentration. Plant Cell Physiol. 2012;53(12):2090-100.

61. Lee, M., Choi, Y., Burla, B., Kim, Y. Y., Jeon, B., Maeshima, M., et al. The $\mathrm{ABC}$ transporter AtABCB14 is a malate importer and modulates stomatal response to $\mathrm{CO}_{2}$. Nat. Cell Biol. 2008;10(10):1217-23.

62. Lin, R., and Wang, H. Two homologous ATP-binding cassette transporter proteins, AtMDR1 and AtPGP1, regulate Arabidopsis photomorphogenesis and root development by mediating polar auxin transport. Plant Physiol. 2005;138(2):949-64.

63. Kaneda, M., Schuetz, M., Lin, B. S. P., Chanis, C., Hamberger, B., Western, T. L., et al. ABC transporters coordinately expressed during lignification of Arabidopsis stems include a set of ABCBs associated with auxin transport. J. Exp. Bot. 2011;62(6):2063-77.

64. Langfelder, P. and Horvath, S. WGCNA: an R package for weighted correlation network analysis. BMC Bioinformatics 2008;9:559.

65. Hawkins, C., and Liu, Z. A model for an early role of auxin in Arabidopsis gynoecium morphogenesis. Front. Plant Sci. 2014;5:327.

66. Yang, C., Wang, D., Zhang, C., Kong, N., Ma, H., and Chen, Q. Comparative analysis of the PIN auxin transporter gene family in different plant species: a focus on structural and expression profiling of PINs in Solanum tuberosum. Int. J. Mol. Sci. 2019;20(13):3270.

67. Lomax, T. L. Auxin transport. Plant Hormones 1995;51(4):494-500.

68. Zhang, K. X., Xu, H. H., Yuan, T. T., Zhang, L., and Lu, Y. T. Blue-light-induced PIN3 polarization for root negative phototropic response in Arabidopsis. Plant J. 2013;76(2):308-21.

69. Mehrotra, R., Bhalothia, P., Bansal, P., Basantani, M. K., Bharti, V., and Mehrotra, S. Abscisic acid and abiotic stress tolerance-different tiers of regulation. J. Plant Physiol. 2014;171(7):486-96.

70. Kemal, K. Auxin and the integration of environmental signals into plant root development. Ann. Bot. 2013;112(9):1655-65. 
71. Wang, Y., Li, K., and Li, X. Auxin redistribution modulates plastic development of root system architecture under salt stress in Arabidopsis thaliana. J. Plant Physiol. 2009;166(15):1637-45.

72. Sun, F., Zhang, W., Hu, H., Li,B., Wang, Y., Zhao,Y., et al. Salt modulates gravity signaling pathway to regulate growth direction of primary roots in Arabidopsis. Plant Physiol. 2008;146(1):178-88.

73. Terasaka, K., Blakeslee, J. J., Titapiwatanakun, B., Peer, W. A., Bandyopadhyay, A., Makam, S. N., et al. PGP4, an ATP binding cassette p-glycoprotein, catalyzes auxin transport in Arabidopsis thaliana roots. Plant Cell 2005;17(11):2922-39.

74. Siepel, A., and Arbiza, L. Cis-regulatory elements and human evolution. Curr. Opin. Genet. Dev. 2014;29:81-9.

75. Weirauch, M. T., and Hughes, T. R. Conserved expression without conserved regulatory sequence: the more things change, the more they stay the same. Trends Genet. 2010;26(2):66-74.

76. Yamaguchi-Shinozaki, K., and Shinozaki, K. Organization of cis-acting regulatory elements in osmotic-and cold-stress-responsive promoters. Trends Plant Sci. 2005;10(2):88-94.

77. Buell, C. R. Michigan State University PGSC. Available online: http://solanaceae.plantbiology.msu.edu (accessed on 2 January 2017).

78. Deng, W., Wang, Y., Liu, Z., Cheng, H., and Xue, Y. HemI: a toolkit for illustrating heatmaps. PLoS One 2014;9(11), e111988.

79. Li, G., Zhou, Y., Zhao, Y., Liu, Y., Ke, Y. et al. Internal reference gene selection for quantitative real-time RT-PCR normalization in potato tissues. PHYTON-INT J EXP BOT 2020;89(2):329-44.

80. Livak, K. J., and Schmittgen, T. D. Analysis of relative gene expression data using real-time quantitative PCR and the $2^{-\Delta \Delta C T}$ method. Methods 2001;25(4):402-08. 

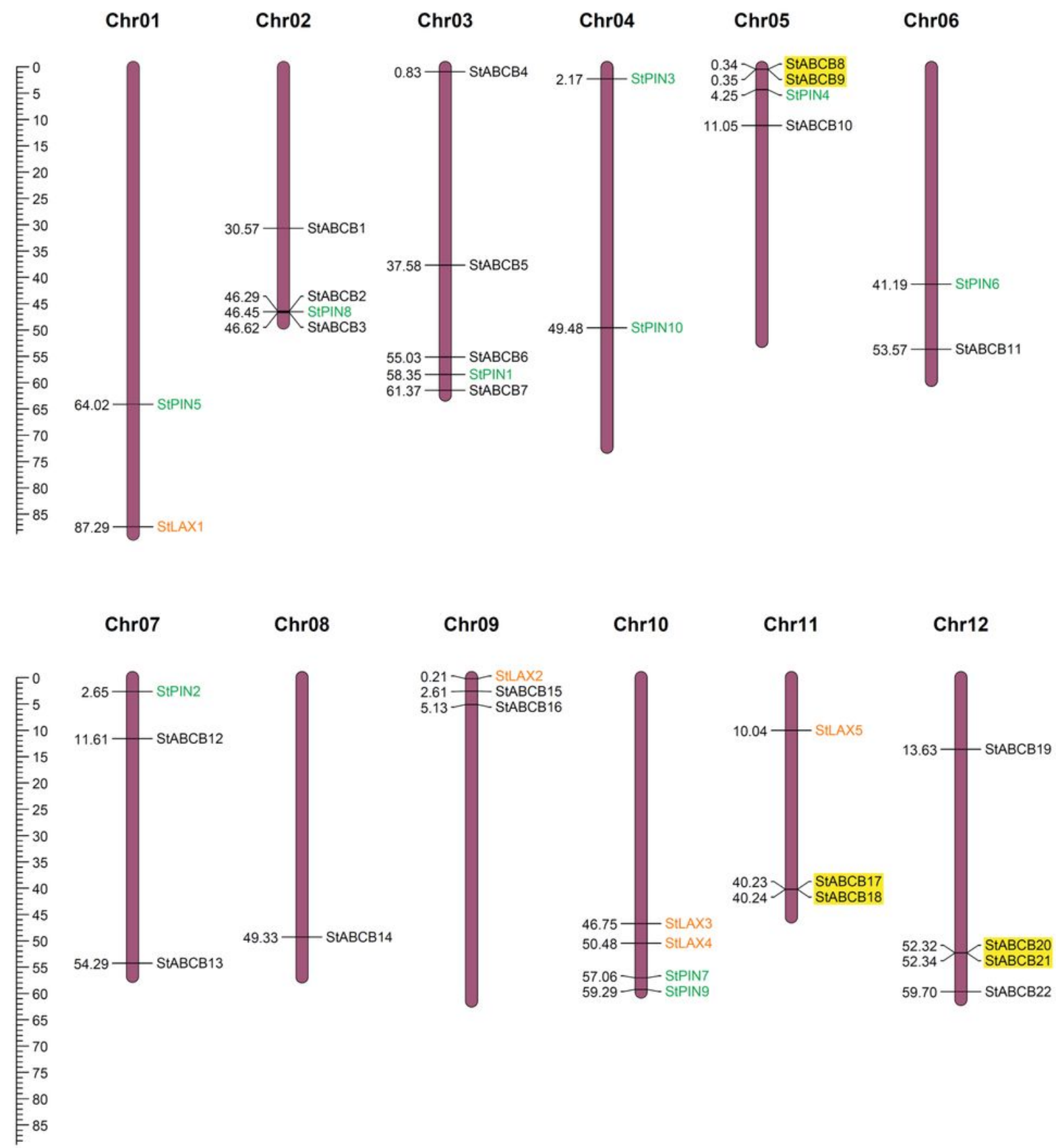

\section{Figure 1}

Chromosomal distribution of StLAX, StPIN, and 485 StABCB family genes. Potato chromosomes were arranged in blocks. Five StLAX genes, 10 StPIN genes and 22 StABCB genes were mapped by locus and the gene clusters were represented by yellow rectangles. 
A

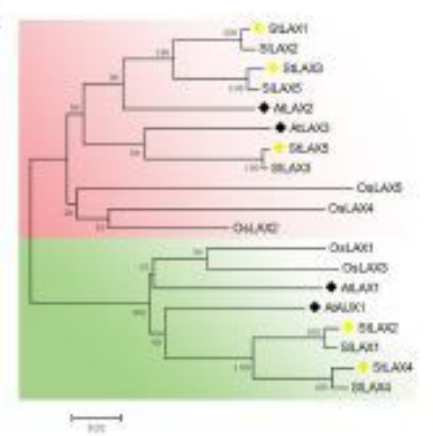

c

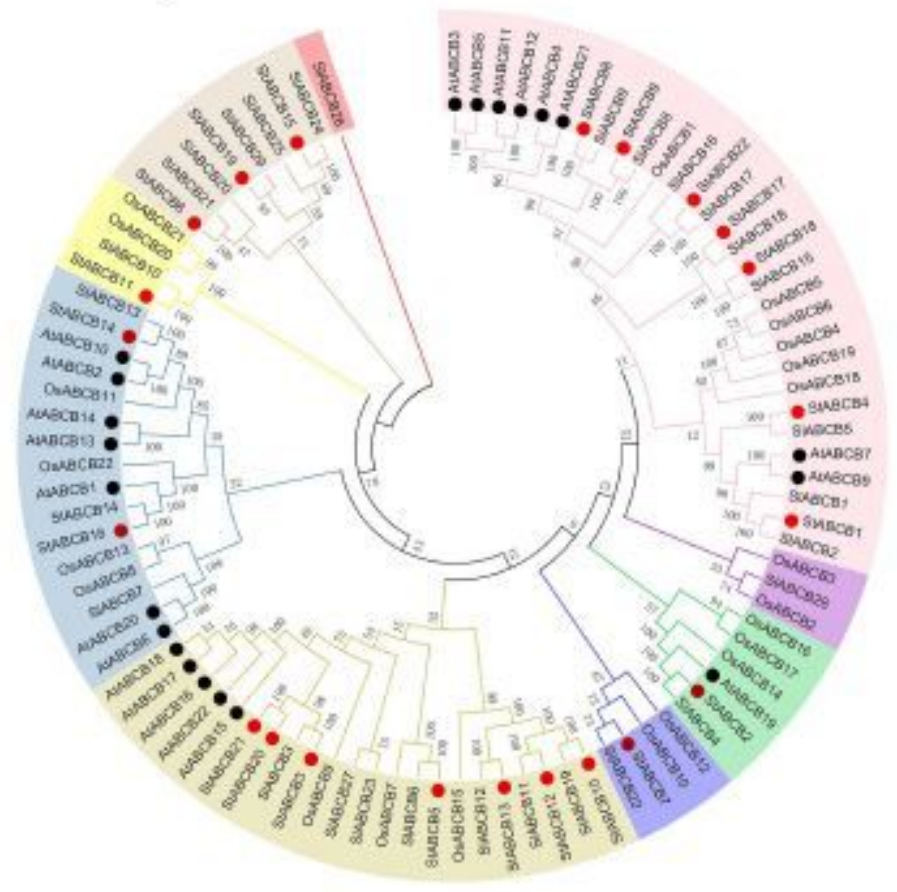

B

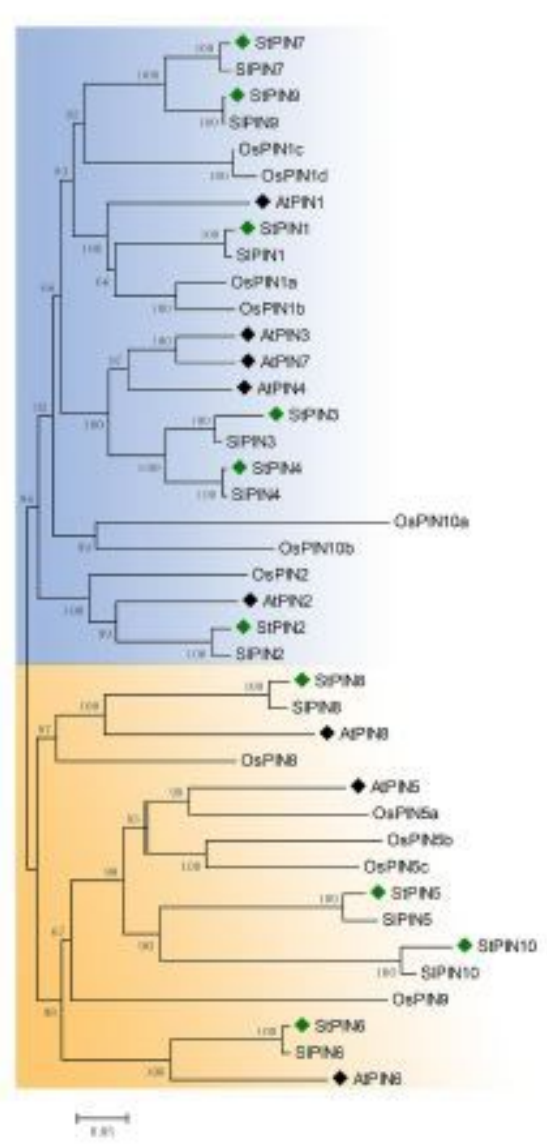

\section{Figure 2}

Phylogenetic tree of LAXs (A), PINs (B), and ABCBs 567 (C) auxin transporter protein families in Arabidopsis, rice, tomato and potato. Bootstrap values were presented for all branches. (A) LAX protein family: inventory of AtLAX families was based on TAIR databases. (B) PIN protein family: sequence data on AtPIN and StPIN families was based on TAIR annotation and Efstathios R's publication[53]. (C) ABCB protein family: inventory of $A t A B C B$ families was based on the $A B C$ superfamily reviewed by Verrier et al[12]. Different colors indicated different subfamilies. 

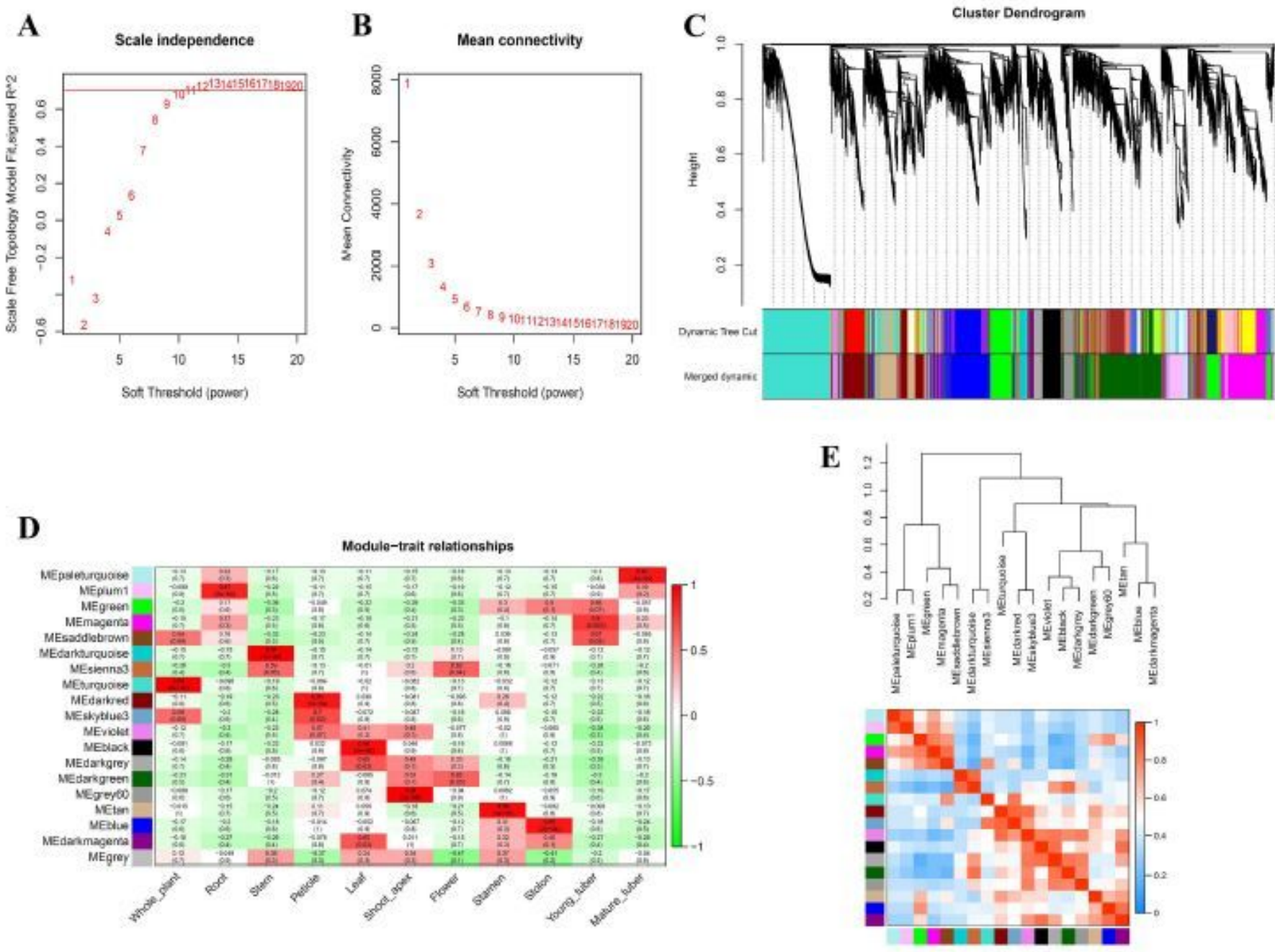

\section{Figure 3}

Graphical visualization of the S. tuberosum co-expression 613 network. (A) Plot showing the scale free topology R2 value in function of increasing soft thresholding power. (B) Plot showing the relation between mean connectivity and soft threshold. (C) Dendrograms produced by average linkage of hierarchical clustering of S. tuberosum genes, which based on a topological overlap matrix (TOM). The modules were assigned colors as indicated in the horizontal bar beneath the dendrogram. (D) Characterizing consensus modules by differential expression of their corresponding eigengenes in the various tissues from potato plant. Red meant over-expression, green meant under-expression; numbers in each cell gave the corresponding t-test p-value. Each column corresponded to a tissue and each row corresponded to an eigengene. (E) Clustering dendrograms of consensus module eigengenes for identifying meta-modules (above) and the heatmap for the correlation coefficient between the modules (below). The diagonal plots showed heatmap plots of eigengene adjacencies. Each row and column corresponded to one eigengene (labeled by consensus module color). Within the heatmap, red indicated high adjacency (positive correlation) and green low adjacency (negative correlation) as shown by the color legend. 


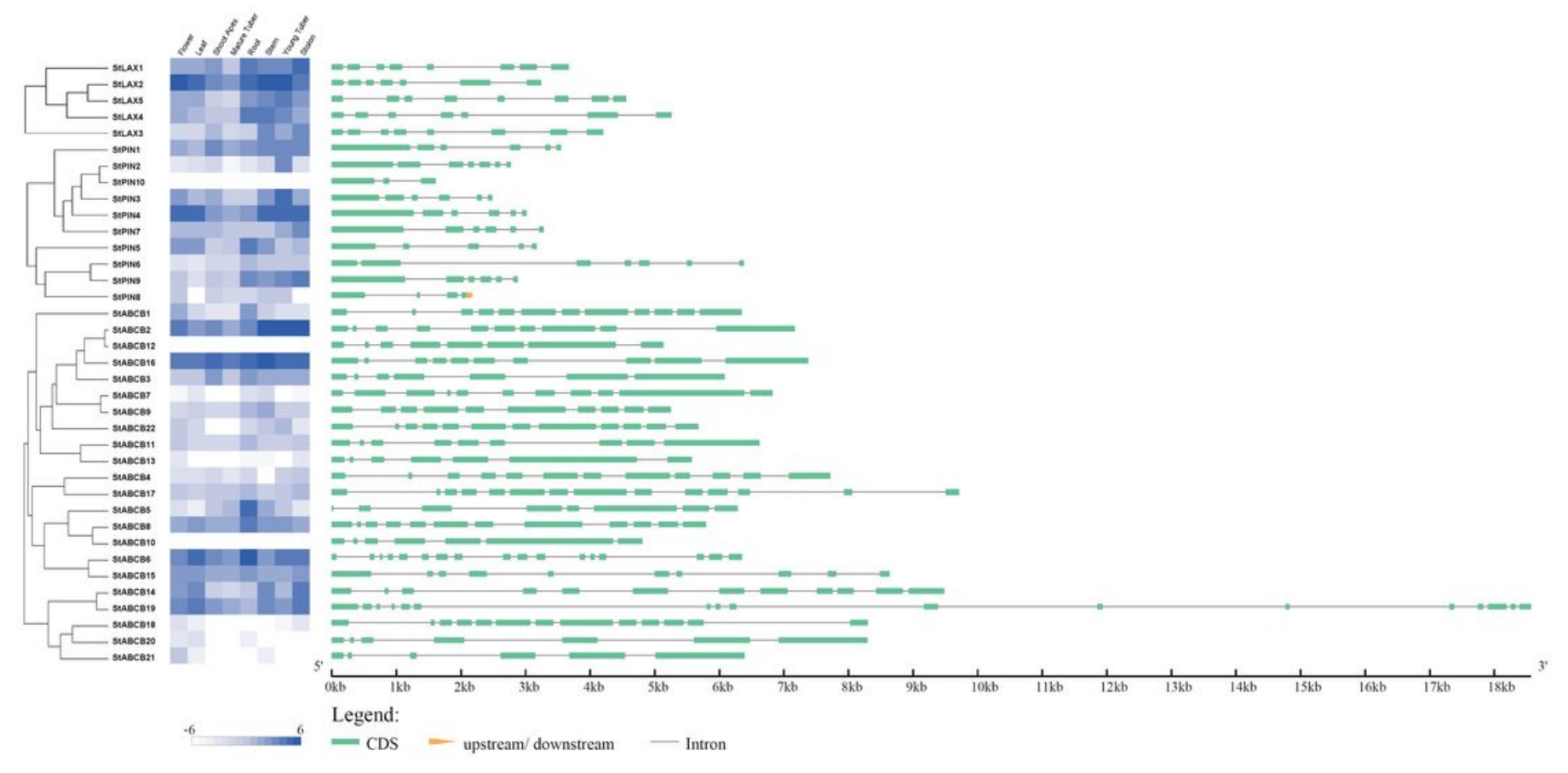

Figure 4

Tissues-specific expressions and exon-intron structures of StLAX, StPIN, and StABCB genes. The heat map was generated using the Cluster 3.0 software according to the RNA-seq data of $\mathrm{RH}$. The exons were indicated by green boxes and the introns were indicated by gray lines.

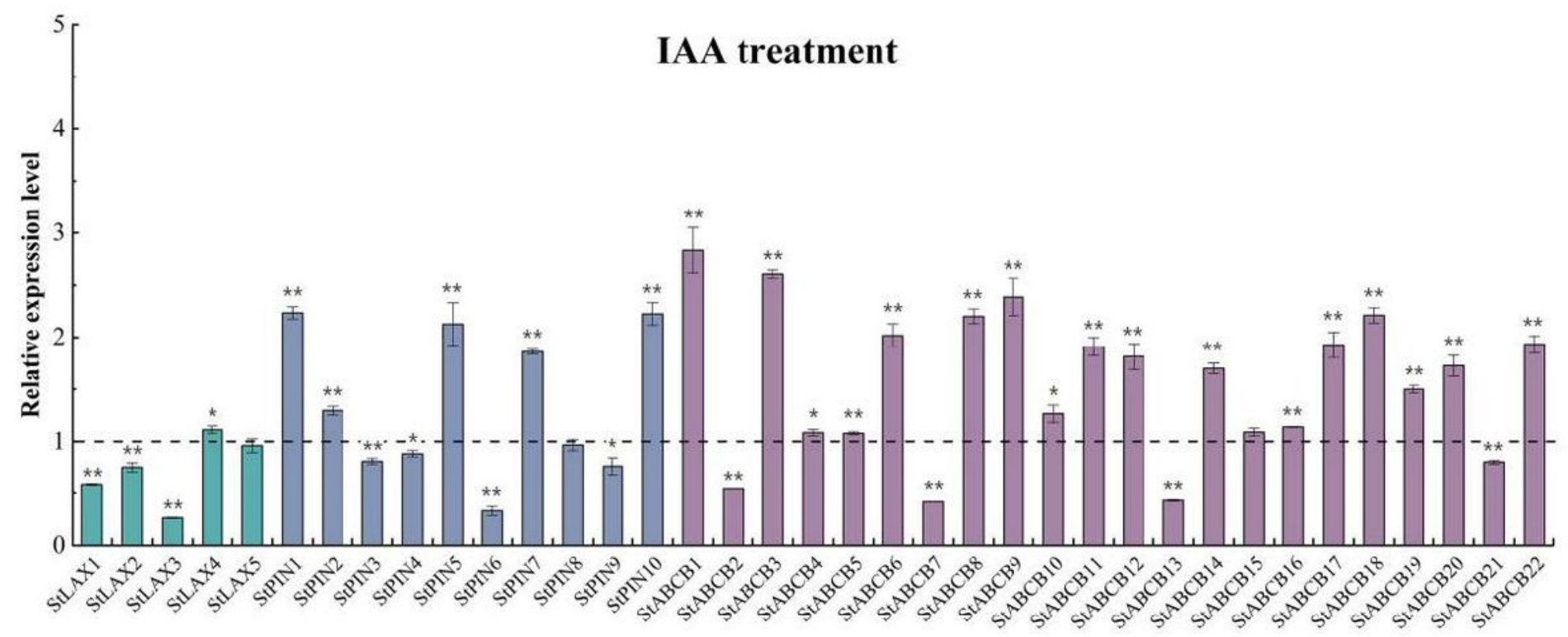

\section{Figure 5}

The relative expression values of auxin transporter StLAX, StPIN, and StABCB genes following IAA treatment. Total RNA was extracted from 4-week-old potato plantlets for expression analysis. The 
histogram represents the relative RNA level of genes after IAA treatment compared with the mock expression level, which was normalized to a value of 1 . The elongation factor 1-a (ef1-a) was employed as the internal standard to normalize the relative mRNA level of individual genes. Error bars represent the standard deviations (SDs) from three biological replicates. ( ${ }^{\star}$ t-test P-value $<0.05$, $* \star$ t-test $P$-value $<0.01$ ).
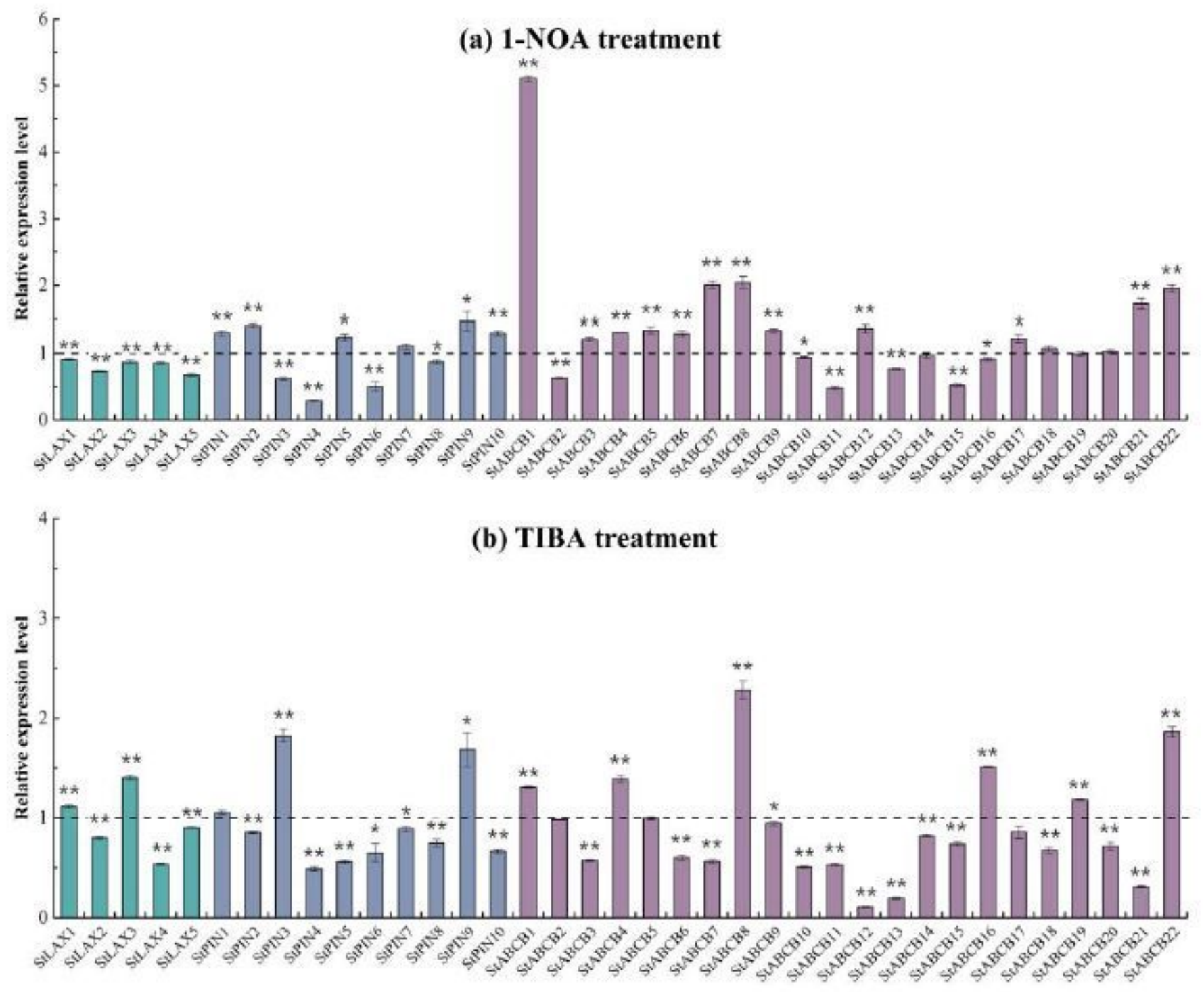

Figure 6

Expression profiles analysis of auxin transporter 766 genes StLAX, StPIN, and StABCB under auxin

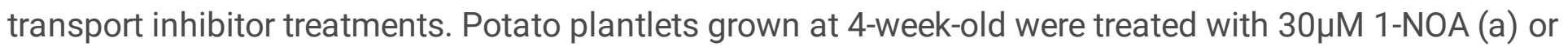
$50 \mu \mathrm{M}$ TIBA (b) for $3 \mathrm{~h}$. The relative expression levels were normalized to a value of 1 in the untreated seedlings. Assays were run in triplicates, and bars represent SDs. ( ${ }^{*}$-test P-value $<0.05,{ }^{*} t$-test $P$-value $<0.01)$. 

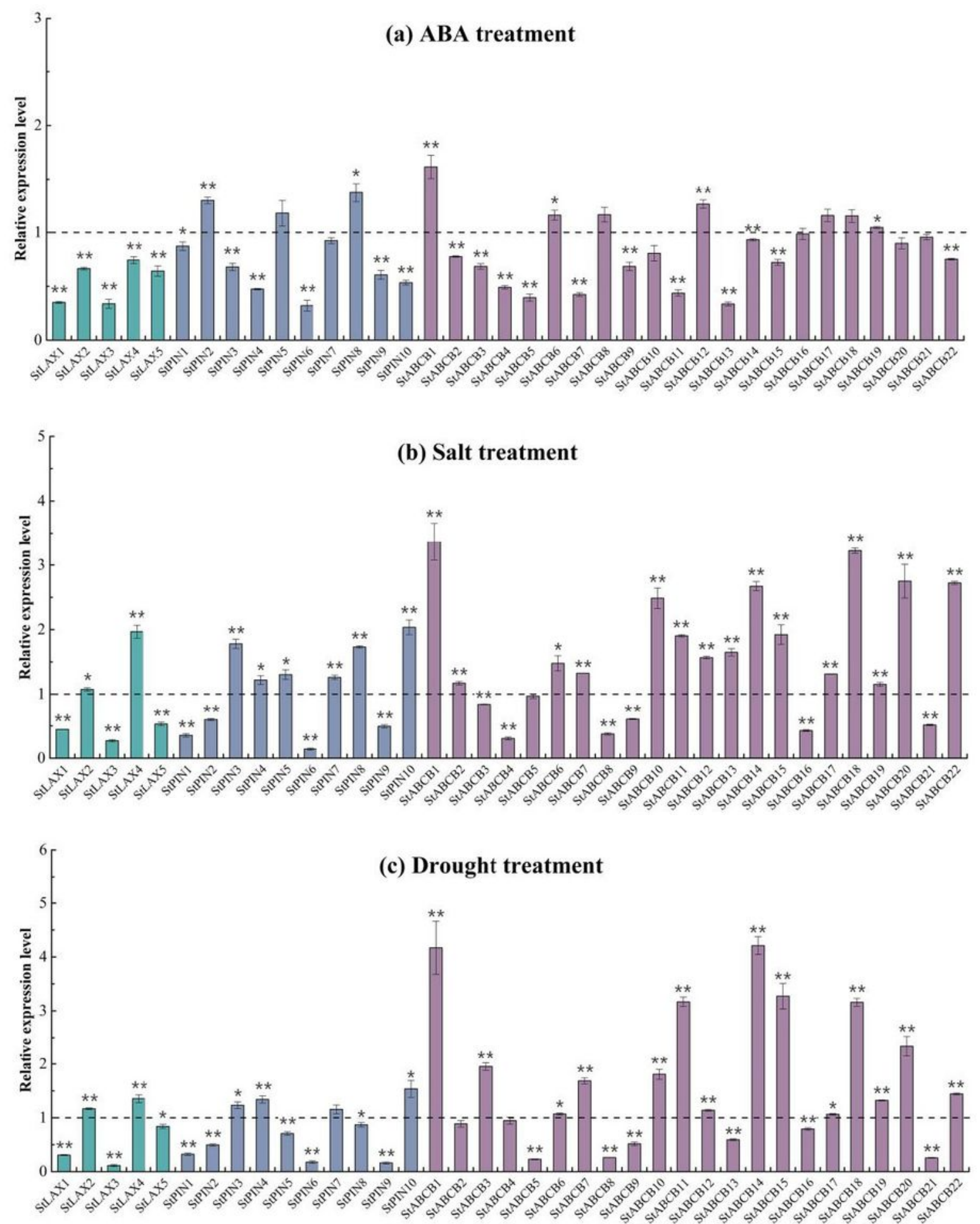

\section{Figure 7}

Expression levels of StLAX, StPIN, and StABCB family 828 genes in response to ABA and abiotic stress. Total RNA was extracted from 4-week-old potato plantlets treated with $100 \mu \mathrm{M}$ ABA (a) for $3 \mathrm{~h}, 200 \mathrm{mM}$ $\mathrm{NaCl}$ (b) or $20 \%$ (W/V) PEG (drought) (c) for 24h. The relative mRNA level of each gene was normalized with respect to the internal reference gene (ef1-a). The data were analyzed by three biological repeats, and SDs were shown with error bars. ( ${ }^{\star} \mathrm{t}$-test $\mathrm{P}$-value $<0.05$, $\star \star t$-test $\mathrm{P}$-value $<0.01$ ). 


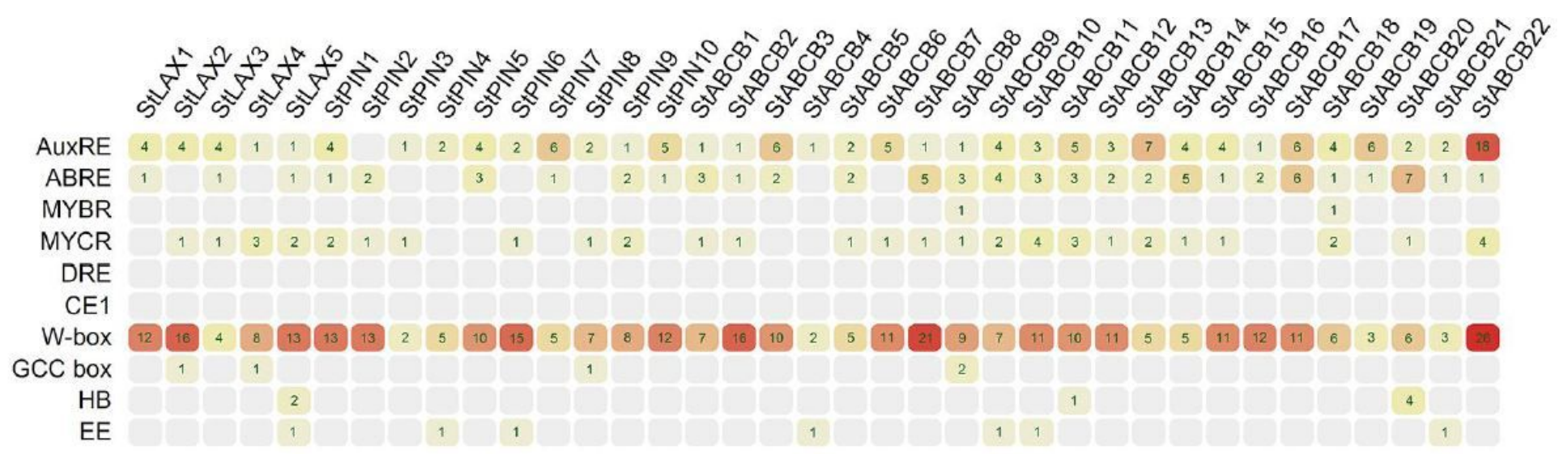

\section{Figure 8}

Analysis of auxin-responsive and stress-related cis-870 regulatory elements in the 2-kb promoter regions of StLAX, StPIN, and StABCB genes.

\section{Supplementary Files}

This is a list of supplementary files associated with this preprint. Click to download.

- Table1.xlsx

- FigureS1.tif

- TableS1.docx

- Tables10.docx

- Tables2.docx

- TableS3.xIsx

- TableS4.docx

- TableS5.xIsx

- TableS6.xlsx

- TableS7.xIsx

- TableS8.xIsx

- TableS9.xIsx 